\title{
Stabilized updated Lagrangian corrected SPH for explicit dynamic problems
}

\author{
Y. Vidal ${ }^{1}$, J. Bonet ${ }^{2}$ and A. Huerta ${ }^{1, *}$ \\ 1 Departament de Matemàtica Aplicada III, Laboratori de Càlcul Numèric (LaCàN), Universitat Politècnica \\ de Catalunya, Jordi Girona 1, E-08034 Barcelona, Spain \\ e-mail: \{yolanda.vidal,antonio.huerta\}@upc.es, web http://www-lacan.upc.es \\ 2 Civil and Computational Engineering Center ( $\left.C^{2} E C\right)$, University of Wales Swansea \\ Singleton Park, Swansea, SA2 8PP, United Kingdom \\ e-mail: j.bonet@swansea.ac.uk
}

\begin{abstract}
SUMMARY
Smooth Particle Hydrodynamics with a total Lagrangian formulation are, in general, more robust than finite elements for large distortion problems. Nevertheless, updating the reference configuration may still be necessary in some problems involving extremely large distortions. However, as discussed here a standard updated formulation suffers the presence of zero energy modes that are activated and may spoil completely the solution. It is important to note that, unlike an Eulerian formulation, the updated Lagrangian does not present tension instability but only zero energy modes. Here an stabilization technique is incorporated to the updated formulation to obtain an improved method without mechanisms and capable to solve problems with extremely large distortions.
\end{abstract}

KEY WORDS: corrected smooth particle hydrodynamics; SPH; meshfree methods; updated Lagrangian formulation; large distortions.

\section{INTRODUCTION}

Corrected Smooth Particle Hydrodynamics (SPH) techniques are nowadays commonly used in some fast-transient dynamics problems. In fact, SPH may be competitive compared to finite elements (FE) because of its low computational cost, its reasonable precision and stability compared with classical methods and, more importantly, its ability to handle large distortions. Nevertheless in the absence of large distortions FE computations are preferred by practitioners

\footnotetext{
* Correspondence to: Departament de Matemàtica Aplicada III, E.T.S. Ingenieros de Caminos, Laboratori de Càlcul Numèric (LaCàN), Universitat Politècnica de Catalunya, Jordi Girona 1, E-08034 Barcelona, Spain.

Contract/grant sponsor: Ministerio de Ciencia y Tecnología; contract/grant number: DPI2004-03000

Contract/grant sponsor: Generalitat de Catalunya AGAUR; contract/grant number: 2003BEAI200248
} 
for two main reasons. First, users are more familiar with FE methods and SPH is still seen a research topic (although this is rapidly changing). Second, FE computations, in the absence of large distortions (and thus in the absence of remeshing), may be very efficient and, thus, less costly (for a given precision) than SPH. However, these advantages disappear in the presence of intensive remeshing. In these cases SPH goes beyond FE methods. Therefore, several authors have proposed to couple FE and SPH which seems a reasonable approach to benefit from the advantages of both formulations see for instance $[1,2,3,4,5,6]$. In any case, for very large distortions even SPH needs to recompute the neighboring particles. This paper focusses only on SPH and its applicability to solve extremely large distortion problems.

In its original form SPH had several weak points, described in detail in [7, 8], cf. also [9] for a review. These problems consisted on lack of consistency, tension instability and the presence of zero energy modes in the numeric solution.

The correction of SPH in order to reproduce polynomials in finite domains as well as passing the patch test has been an area of intensive work. Some of these contributions, without being exhaustive, are discussed in this paper. See [10] for a detailed discussion on reproducibility of SPH methods or [9] for a general review of meshfree methods. Some of these techniques are the normalized smoothing method proposed in [11,3] for obtaining linear consistency; a corrected kernel (invoking a Taylor series expansion) introduced in [12]; the Corrected Smooth Particle Hydrodynamics method that allows to obtain linear consistency in the interpolation of the function and in the interpolation of the gradient developed in [13] (consistency is achieved introducing corrections in the kernel functions and in their derivatives); or the stabilized
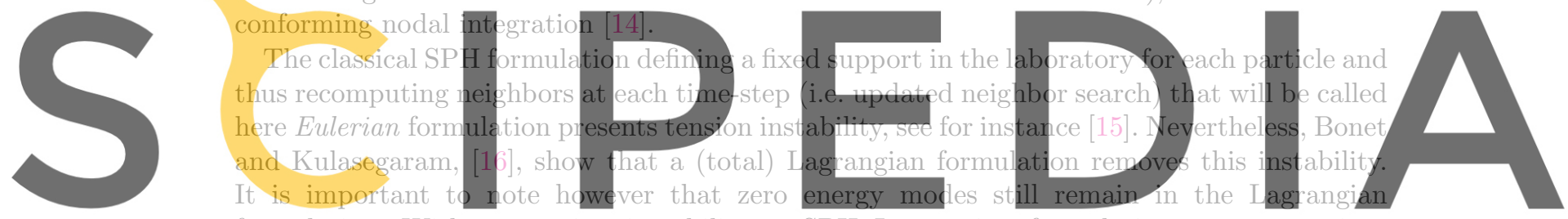

formulation. Without tension instability, a SPH Lagrangian formulation presents serious

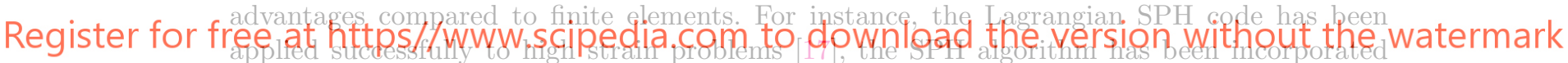

into a standard Lagrangian code such as EPIC [18], and it has also been used for impact problems $[19,3]$. Nevertheless, in problems with severe distortions a Lagrangian formulation will still require updates of the reference configuration. When such updates are incorporated zero energy modes are more likely to be activated. When few updates are performed during the computation the induced errors may remain unnoticed. But when updates are performed frequently the solution is completely spoilt, because zero energy modes are excited and they produce spurious oscillations. The objective of this paper is to develop an updated Lagrangian formulation that can carry out updates of the reference configuration without suffering from spurious modes.

The problem of zero energy modes is still open. In the literature two types of solutions are used: dissipation of spurious modes (conceptually similar to the techniques used in finite elements for hourglass modes) or an alternative discretization that does not evaluate the variables and their derivatives at the same points. For example, in [20] instability is precluded introducing an artificial stress (but that introduces also small errors in the solution) and in $[21,22]$ different sets of particles are used to interpolate different fields generating the denominated stress points. Here, for computational efficiency, particles are the only information 

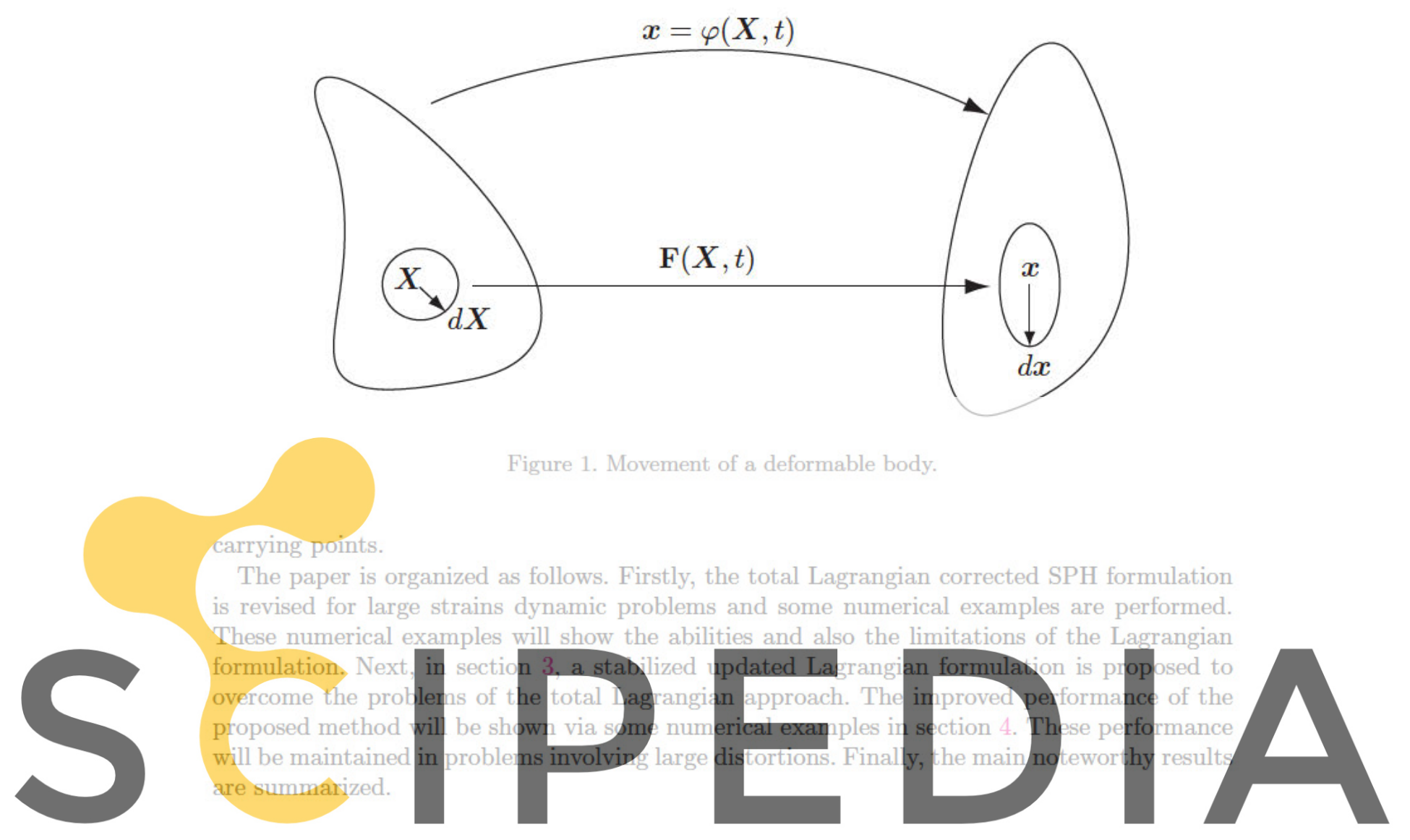

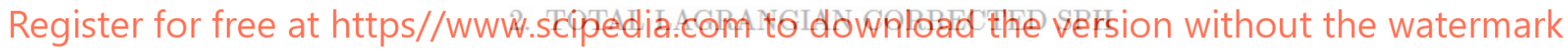

\subsection{Continuum equations}

Consider the three-dimensional continuum shown in Figure 1 undergoing a given motion defined by a mapping $\psi$ between initial and current positions as,

$$
x=\varphi(X, t) .
$$

The deformation gradient $\mathbf{F}$, defined as

$$
\mathbf{F}=\frac{\partial x}{\partial \boldsymbol{X}},
$$

is a quantity of interest in the study of large deformations because it is present in all those equations that relate magnitudes in the initial configuration with their corresponding ones in the final configuration. The volume change of the continuum can be obtained in terms of the Jacobian

$$
J=\operatorname{det} \mathbf{F}=\frac{d V}{d V^{0}},
$$


where $d V^{0}$ and $d V$ represent the initial and current element volumes. The momentum balance equation for the deformable body reads

$$
\nabla \cdot \sigma+\zeta f=\zeta a,
$$

where $\zeta$ is the final density of the material, $\boldsymbol{a}$ is the acceleration, $\boldsymbol{f}$ is external force for unit deformed volume and $\boldsymbol{\sigma}$ is the Cauchy stress tensor. Recall that,

$$
\mathbf{P}=J \sigma \mathbf{F}^{-\top}
$$

where $\mathbf{P}$ is the first Piola-Kirchhoff tensor. The equilibrium equation can also be expressed in the reference (Lagrangian) configuration in terms of $\mathbf{P}$ and the density in the reference configuration $\zeta_{0}$ as, see [23],

$$
\nabla_{0} \cdot \mathbf{P}+\zeta_{0} f_{0}=\zeta_{0} a .
$$

where $f_{0}$ is external force for unit undeformed volume. Alternatively, equilibrium can be expressed by means of the principle of virtual work expressed in the reference configuration as

$$
\int_{V^{0}} \zeta_{0} \boldsymbol{a} \cdot \delta \mathbf{v} d V^{0}+\int_{V^{0}} \mathbf{P}: \delta \dot{\mathbf{F}} d V^{0}=\int_{V^{0}} \boldsymbol{f}_{0} \cdot \delta \mathbf{v} d V^{0}+\int_{\partial V^{0}} \mathrm{t}_{0} \cdot \delta \mathbf{v} d A^{0}
$$

where $\delta \mathbf{v}$ denotes an arbitrary virtual velocity from the current position of the body and $\mathbf{t}_{0}$ is the traction vector.

2.2. Corrected SPH approximation
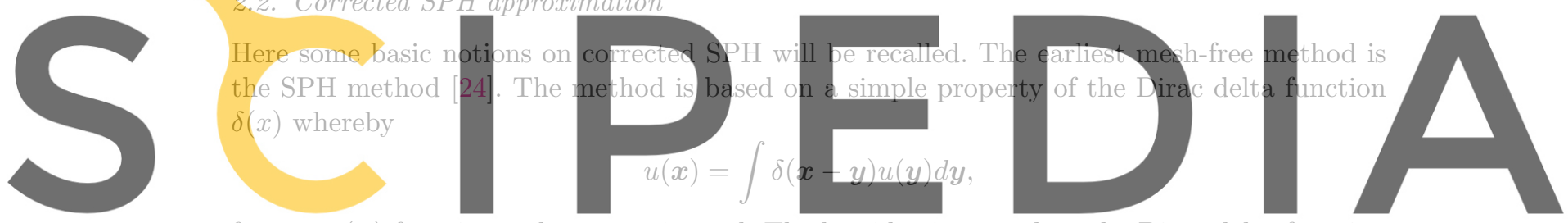

for any $u(x)$ function to be approximated. The key idea is to replace the Dirac delta function

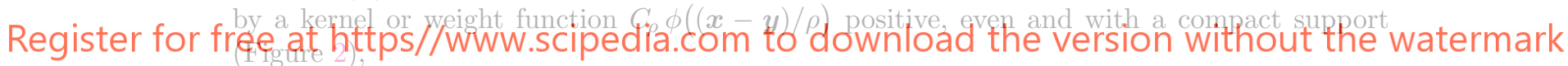

$$
u(x) \simeq \widetilde{u}^{\rho}(x):=\int C_{\rho} \phi\left(\frac{x-y}{\rho}\right) u(y) d y,
$$

where $\rho$ is called the dilation parameter and is usually the support radius of the kernel function. $C_{\rho}$ is a normalization constant such that

$$
\int C_{\rho} \phi\left(\frac{\boldsymbol{x}-\boldsymbol{y}}{\rho}\right) d \boldsymbol{y}=1
$$

i.e. constant functions are exactly interpolated. Therefore, as $\rho$ tends to zero the kernel function approaches the Dirac delta function, and consequently,

$$
\lim _{\rho \rightarrow 0} \widetilde{u}^{\rho}(\boldsymbol{x})=u(\boldsymbol{x}) .
$$

In order to develop a computational technique, it is necessary to evaluate the integration in equation (4) in a discrete manner to give

$$
u(\boldsymbol{x}) \simeq \widetilde{u}^{\rho}(\boldsymbol{x}) \simeq u^{\rho}(\boldsymbol{x}):=\sum_{i} V_{i} C_{\rho} \phi\left(\frac{\boldsymbol{x}-\boldsymbol{x}_{i}}{\rho}\right) u\left(\boldsymbol{x}_{i}\right),
$$




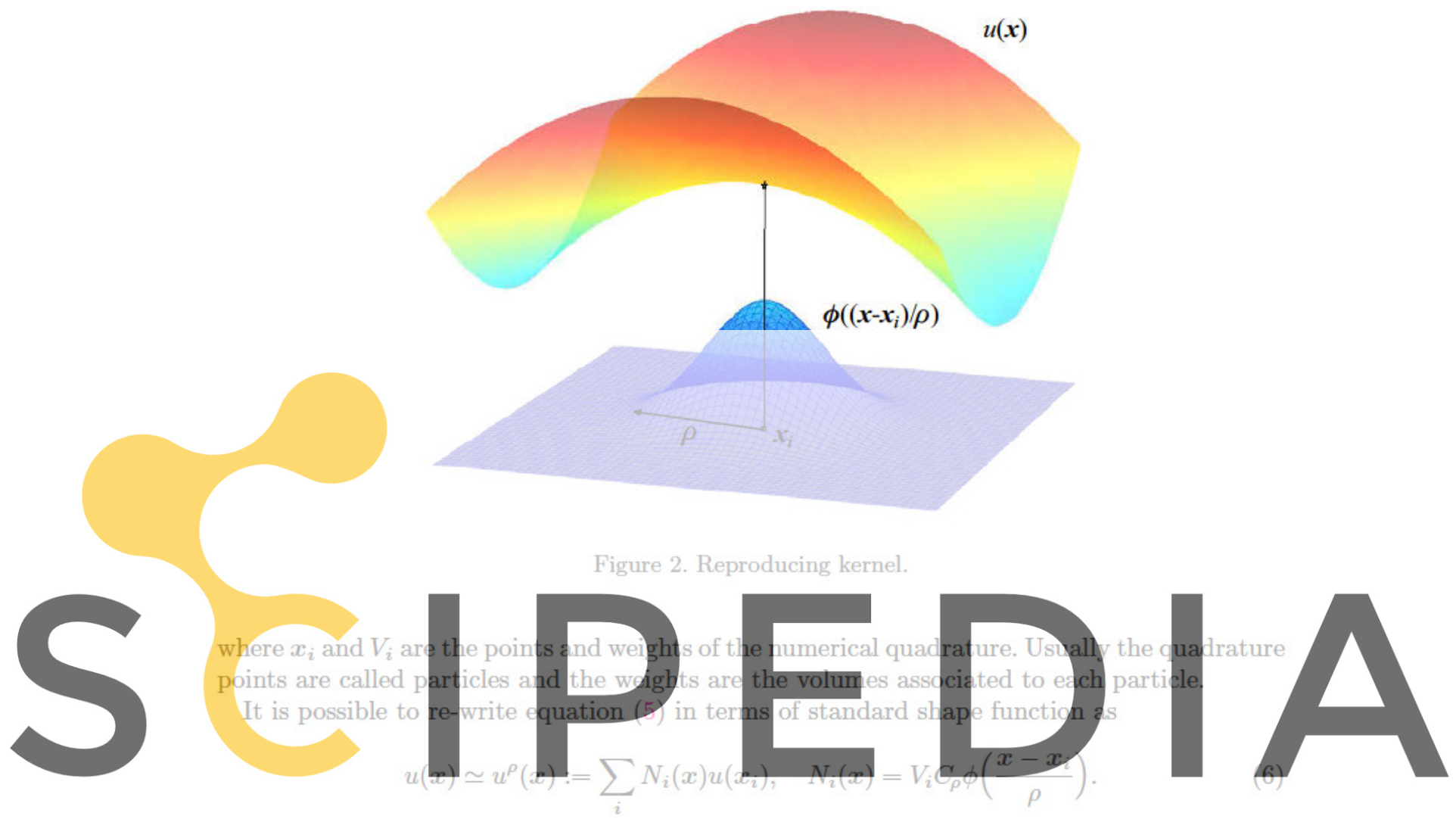

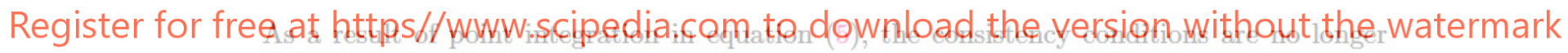
satisfied exactly. Reference [13] presents a corrected SPH approximation to preclude these difficulties. The foregoing is a brief review of the two main corrections introduced by Bonet and coworkers.

Firstly, the aforementioned discrepancy is eliminated by a kernel correction. As proposed in [25], $C_{\rho}$ is selected by enforcing linear consistency conditions now given by a point-wise integration as,

$$
\sum_{i} V_{i} C_{\rho} \phi\left(\frac{x-x_{i}}{\rho}\right)=1, \quad \sum_{i} V_{i}\left(x-x_{i}\right) C_{\rho} \phi\left(\frac{x-x_{i}}{\rho}\right)=0 .
$$

These equations lead to,

$$
C_{\rho}=\alpha(x)\left(1+\beta(x) \cdot\left(x-x_{i}\right)\right)
$$

where

$$
\alpha(x)=\left(\sum_{i} V_{i} \phi\left(\frac{x-x_{i}}{\rho}\right)\left(1+\beta(x) \cdot\left(x-x_{i}\right)\right)\right)^{-1},
$$


and

$$
\boldsymbol{\beta}(\boldsymbol{x})=\left(\sum_{i} V_{i} \phi\left(\frac{\boldsymbol{x}-\boldsymbol{x}_{i}}{\rho}\right)\left(\boldsymbol{x}-\boldsymbol{x}_{i}\right)\left(\boldsymbol{x}-\boldsymbol{x}_{i}\right)^{\top}\right)^{-1} \sum_{i} V_{i}\left(\boldsymbol{x}_{i}-\boldsymbol{x}\right) \phi\left(\frac{\boldsymbol{x}-\boldsymbol{x}_{i}}{\rho}\right) .
$$

The use of this type of correction ensures that linear functions are perfectly interpolated and their gradients are exactly obtained. A possible way of simplifying the calculation is by using constant, rather than linear, correction. This is equivalent to taking $\boldsymbol{\beta}(\boldsymbol{x})=\mathbf{0}$ in equation (7). Nevertheless, gradient evaluation using the above expressions is expensive, both in computer memory and time consuming.

Secondly, the gradient functions are directly amended to ensure that the gradient of a general constant or linear function is correctly evaluated. The corrected gradient is defined as

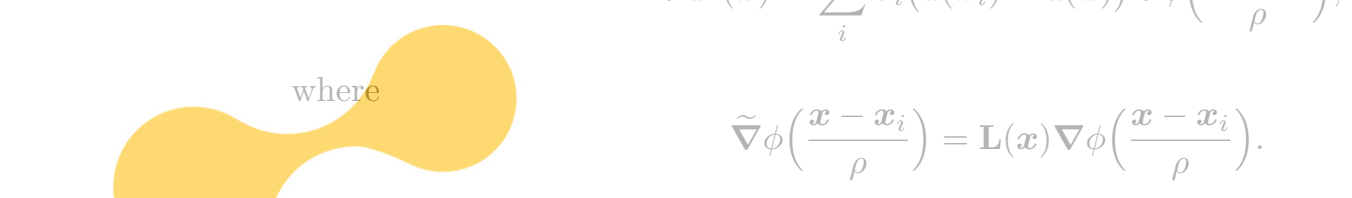

$$
\begin{gathered}
\tilde{\nabla} u^{\rho}(x)=\sum_{i} V_{i}\left(u\left(x_{i}\right)-u(x)\right) \tilde{\nabla} \phi\left(\frac{\boldsymbol{x}-\boldsymbol{x}_{i}}{\rho}\right), \\
\widetilde{\nabla} \phi\left(\frac{x-x_{i}}{\rho}\right)=\mathrm{L}(x) \nabla \phi\left(\frac{x-x_{i}}{\rho}\right) .
\end{gathered}
$$

It is clear that equation (8) will ensure that the gradient of a constant function vanishes. The correction matrix $\mathbf{L}(x)$ is obtained after imposing the linear consistency condition, namely
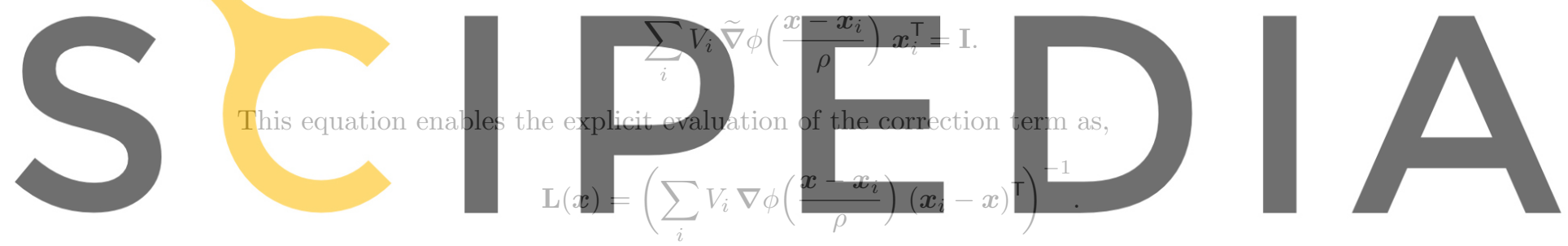

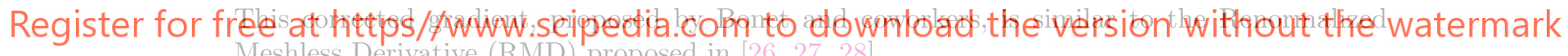
Meshless Derivative (RMD) proposed in [26, 27, 28].

\subsection{Lagrangian corrected $S P H$}

This section will not be devoted to develop or discuss Lagrangian corrected SPH formulation in detail. For an excellent reference see [16]. Here some basic notions will be recalled in order to introduce the notation and the approach employed in following sections.

Let us consider a discretized body using SPH particles. The mapping $\varphi$ between initial and current positions can be approximated using SPH approximation as,

$$
\boldsymbol{x}_{j}=\boldsymbol{\varphi}\left(\boldsymbol{X}_{j}, t\right)=\sum_{k} V_{k} C_{\rho} \phi\left(\frac{\boldsymbol{X}_{j}-\boldsymbol{X}_{k}}{\rho}\right) \boldsymbol{x}_{k} .
$$

The deformation gradient, defined in (1), can be evaluated now in a certain particle $j$ in terms of the current positions just taking derivatives as

$$
\mathbf{F}_{j}=\nabla_{0} \boldsymbol{\varphi}=\sum_{k} \boldsymbol{x}_{k} \mathbf{G}_{k}^{\top}\left(\boldsymbol{X}_{j}\right),
$$


where $\boldsymbol{\nabla}_{0}$ indicates the gradient respect to the initial configuration, $\boldsymbol{x}_{k}$ is the current position of particle $k$ and where the functions $\mathbf{G}$ contain the corrected kernel gradients at the initial configuration, that is,

$$
\mathbf{G}_{k}\left(\boldsymbol{X}_{j}\right)=V_{k} \widetilde{\boldsymbol{\nabla}}_{0} \phi\left(\frac{\boldsymbol{X}_{j}-\boldsymbol{X}_{k}}{\rho}\right),
$$

where $\widetilde{\boldsymbol{\nabla}}_{0}$ is a "corrected" gradient to ensure linear completeness as shown in (8).

In order to find general equations for the internal forces using a Lagrangian corrected SPH formulation, consider the equation of the internal virtual work in the reference configuration in terms of the first Piola-Kirchhoff tensor, $\mathbf{P}$,

$$
\delta \dot{w}_{\text {int }}=\int_{V^{0}} \mathbf{P}: \delta \dot{\mathbf{F}} d V^{0} \simeq \sum_{j} V_{j}^{0} \mathbf{P}_{j}: \delta \dot{\mathbf{F}}_{j} .
$$

The variation of the virtual deformation gradient emerges from equation (9) as

$$
\delta \dot{\mathbf{F}}_{j}=\sum_{k} \delta \mathbf{v}_{k} \mathbf{G}_{k}^{\top}\left(\boldsymbol{X}_{j}\right),
$$

where after substituting into (10) leads to the expression of the internal virtual work

$$
\delta \dot{w}_{\mathrm{int}} \simeq \sum V_{j}^{0} \mathbf{P}_{j}:\left(\sum \delta \mathbf{v}_{k} \mathbf{G}_{k}^{\top}\left(\boldsymbol{X}_{j}\right)\right)=\sum \delta \mathbf{v}_{k} \cdot\left(\sum V_{j}^{0} \mathbf{P}_{j} \mathbf{G}_{k}\left(\boldsymbol{X}_{j}\right)\right) .
$$
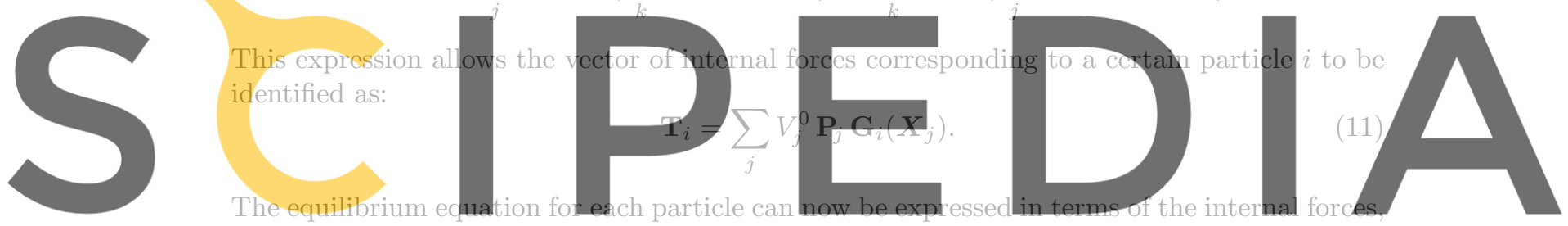

$\mathbb{T}_{i}$, the external forces, $\mathbb{E}_{i}$, and the particle acceleration, $\mathrm{a}_{i}$ in the standard form as [16],

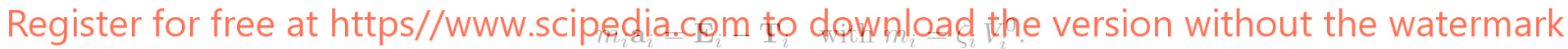

These equations are the integrated using a standard leap-frog scheme [3].

It is important to observe that in (11) the kernel derivatives, $\mathbf{G}_{i}\left(\boldsymbol{X}_{j}\right)$, are fixed in the reference configuration and therefore they do not depend on the current positions of the particles. This implies that corrections are only calculated at the beginning reducing the computational cost.

\subsection{Numerical Examples}

In order to illustrate the ability and limitations of Lagrangian corrected SPH formulation some numerical examples are solved.

2.4.1. Bending test of hyperelastic material. This example, which is very sensible to instabilities, consists in the simulation of a three-dimensional problem with large deformations using a hyperelastic material. This example was also solved in [16]. Consider a nearly incompressible neo-Hookean, see [23], cylinder travelling with an initial speed of $1.88 \mathrm{~m} / \mathrm{s}$ which is suddenly fixed at its base (see Figure 3). Homogeneous Dirichlet boundary conditions 
are directly imposed to the coefficients, which is standard in SPH, because the characteristic radius of the support of the kernel function is small. For larger values of $\rho$ other alternatives may be implemented [29]. The initial radius is $0.32 \mathrm{~m}$ and the length $3.24 \mathrm{~m}$. The shear modulus is taken as $0.3571 \mathrm{MN} / \mathrm{m}^{2}$ and the bulk modulus is $1.67 \mathrm{MN} / \mathrm{m}^{2}$.

The results obtained using a Lagrangian corrected SPH formulation can be seen in Figure 4. The bar oscillates from initial position to maximum deformation and then back to initial position as expected. The stress component $\sigma_{z z}$ is shown where $z$ is the height component. The cylinder deformation is simulated with good results even in the presence of high tension.

2.4.2. Rubber rings collision. This is a classical benchmark test for tension instability, see [20]. This test consists in simulating the collision of two rubber rings coming together at a relative speed of $1.18 \mathrm{~m} / \mathrm{s}$, and then bounce off each other without disintegration. The material is neo-Hookean and hyperelastic as in the previous example. The exterior radius of each ring is $4 \mathrm{~cm}$ and the interior radius is $3 \mathrm{~cm}$. Figure 5 shows how the two rings collide, bounce and then oscillate with no fracture. In fact, calculations were performed only with one rubber ring and symmetry conditions. As expected, the total Lagrangian formulation performs correctly because the relative motion between particles is moderate.

2.4.3. Punch test. Previous examples have been solved using a total Lagrangian approach with very good results. Here an example with extremely large distortions is presented, see $[30,31,6]$. The problem consists in a hyperelastic material equal to the one used in previous
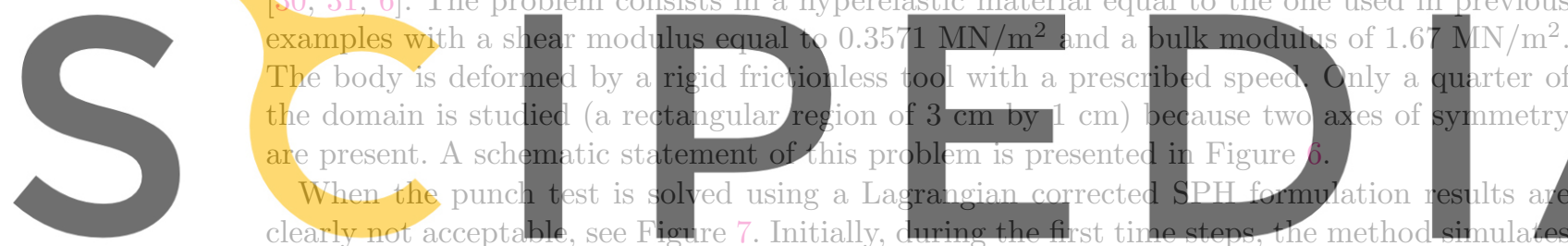

properly the expected behavior, but as the deformation progresses the Lagrangian formulation

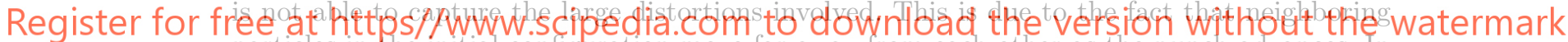

particles in the initial configuration move far away from each other as the punch advances. In

order to circumvent this problem some updates of the reference configuration are necessary

(the neighboring particies must be recomputed). Next section presents a detailed study of such an "updated Lagrangian" formulation.

\section{STABILIZED UPDATED LAGRANGIAN FORMULATION}

Before a stabilized updated Lagrangian formulation is proposed to overcome the problems of the total Lagrangian approach the standard updated Lagrangian one is recalled.

\subsection{Standard updated formulation}

The updated Lagrangian formulation consists of a multiplicative incremental approach as illustrated in Figure 8. An intermediate configuration $\boldsymbol{x}^{r}$ will be used as the new reference configuration for the next time steps. This means that a new neighbor search must be done in configuration $\boldsymbol{x}^{r}$ and that corrections of the kernel and its derivatives must be recalculated. 

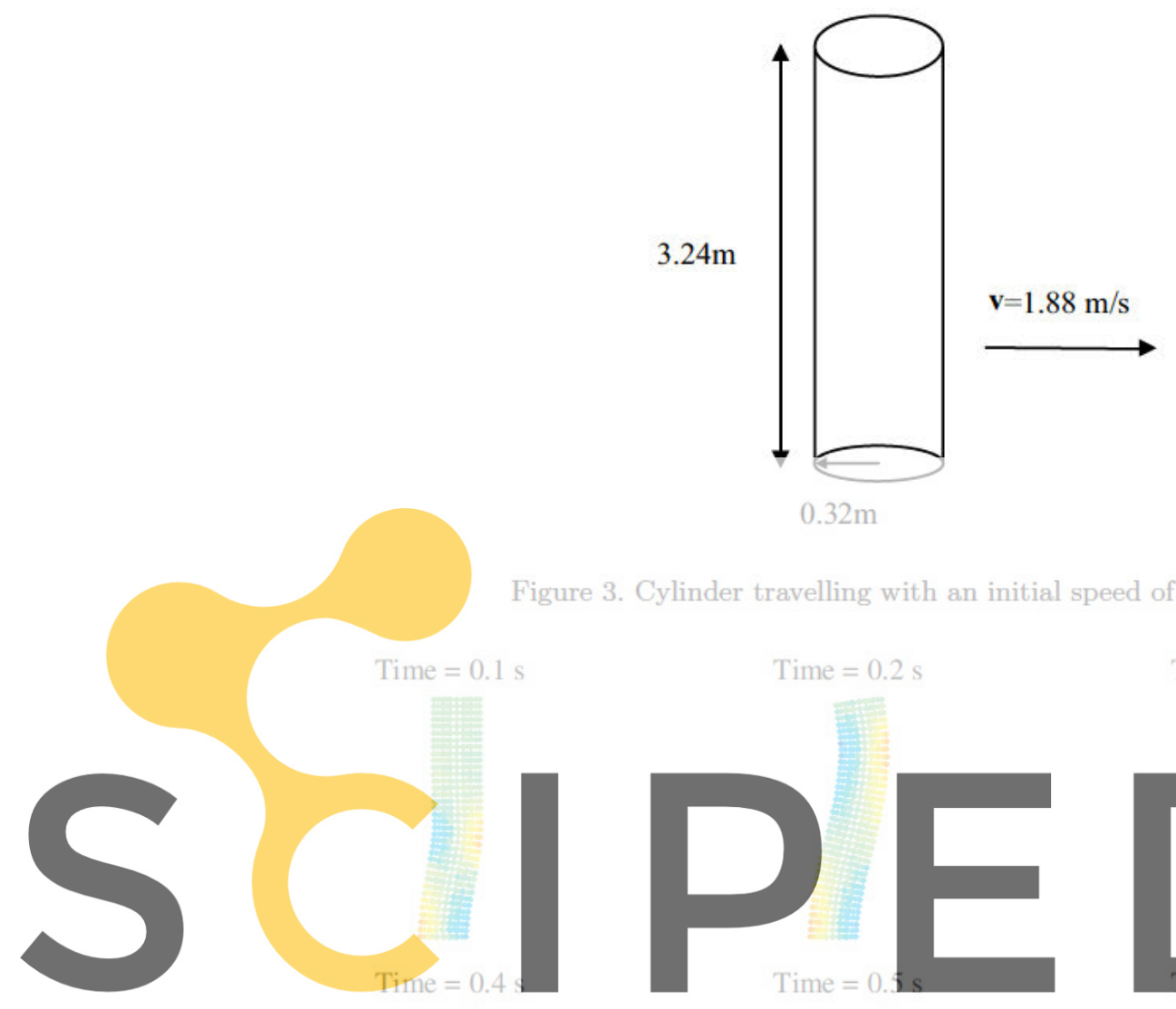

Figure 3. Cylinder travelling with an initial speed of $1.88 \mathrm{~m} / \mathrm{s}$.
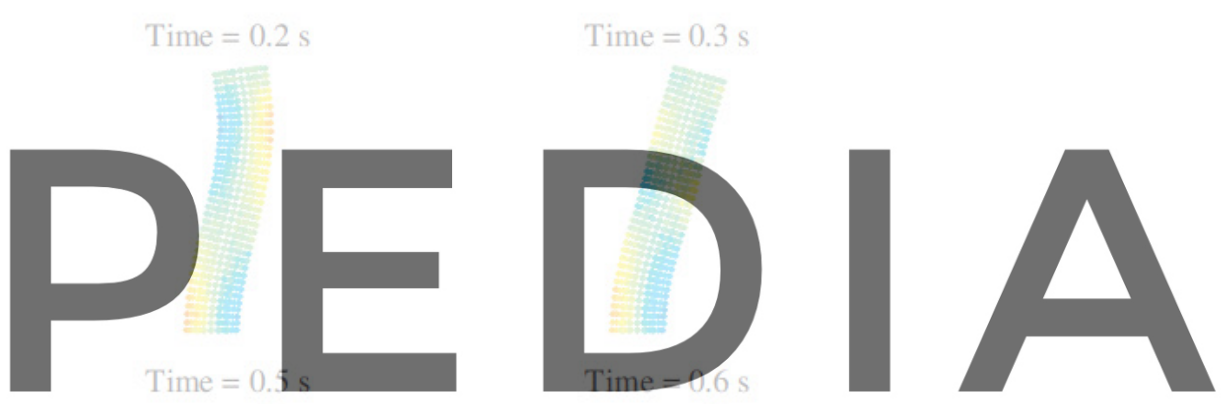

Register for free at https//www.scipedia.com to download the version without the watermark
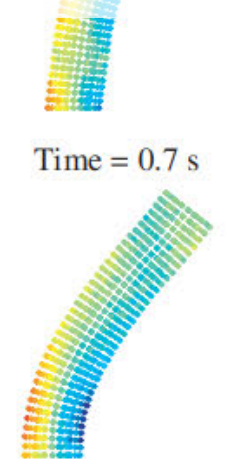

Figure 4. Total Lagrangian formulation: cylinder bending test. Stress component $\sigma_{z z}(z$ is the height component) is shown. 


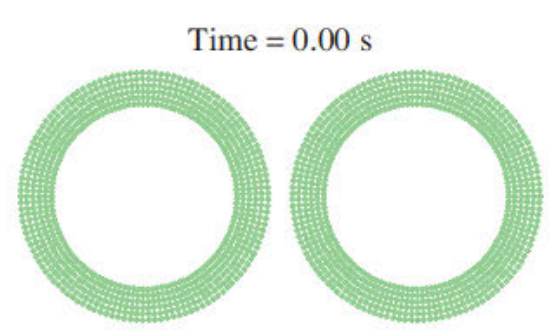

Time $=0.02 \mathrm{~s}$

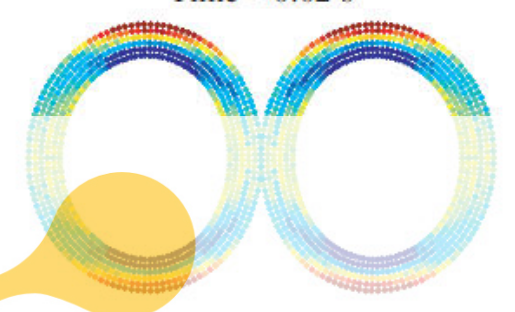

Time $=0.04 \mathrm{~s}$

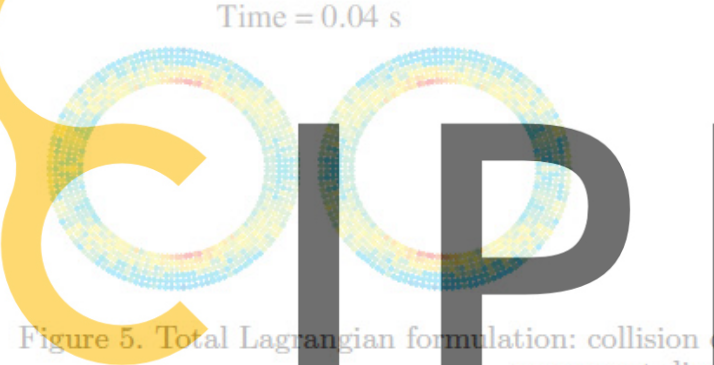

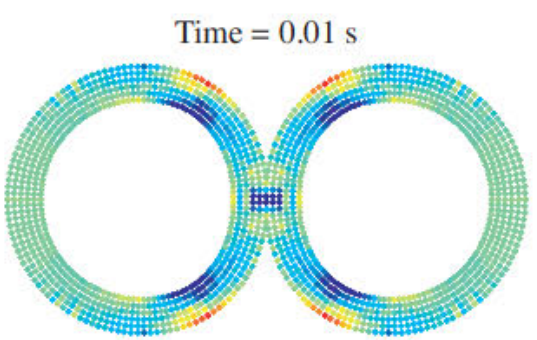

Time $=0.03 \mathrm{~s}$
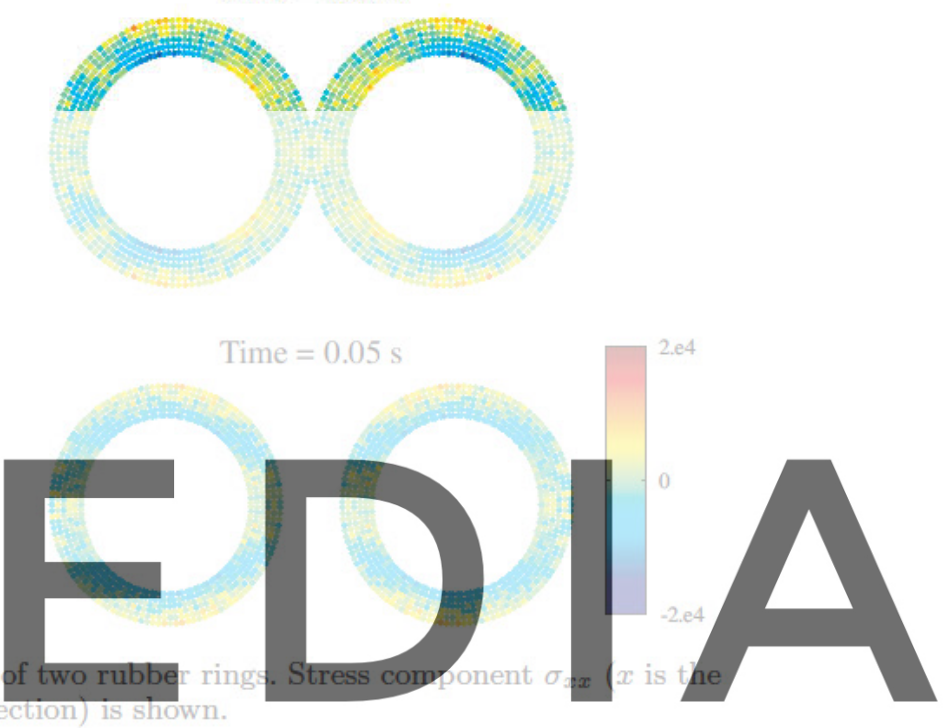

Register for free at https//www.scipedia.com to download the version without the watermark variable and only the incremental deformation gradient, $f$, (i.e. the deformation gradient between the new reference configuration and the final one) is calculated each time step. This update induces minor modification for the evaluation of the internal forces calculation.

The incremental deformation gradient and its variation are written as

$$
\mathbf{f}_{j}=\left.\frac{\partial \boldsymbol{x}^{n}}{\partial \boldsymbol{x}^{r}}\right|_{j}=\sum_{k} \boldsymbol{x}_{k}^{n} \boldsymbol{g}_{k}^{\top}\left(\boldsymbol{x}_{j}^{r}\right), \quad \text { and } \quad \delta \dot{\mathbf{f}}_{j}=\sum_{k} \delta \mathbf{v}_{k} \boldsymbol{g}_{k}^{\top}\left(\boldsymbol{x}_{j}^{r}\right)
$$

where the corrected kernel gradients in the new reference configuration can be defined as

$$
\boldsymbol{g}_{k}\left(\boldsymbol{x}_{j}^{r}\right):=V_{k}^{r}\left[\tilde{\boldsymbol{\nabla}}_{r} \phi\left(\frac{\boldsymbol{x}_{j}^{r}-\boldsymbol{x}_{k}^{r}}{\rho}\right)\right] .
$$

Thus, the variation of internal virtual work expressed in the initial configuration is

$$
\delta \dot{w}_{\text {int }}=\int_{V^{0}} \mathbf{P}^{n}: \delta \dot{\mathbf{F}}^{n} d V^{0} \simeq \sum_{j} V_{j}^{0} \mathbf{P}_{j}^{n}: \delta \dot{\mathbf{F}}_{j}^{n}=\sum_{j} V_{j}^{0} \mathbf{P}_{j}^{n}:\left(\delta \dot{\mathbf{f}}_{j} \mathbf{F}_{j}^{r}\right) .
$$




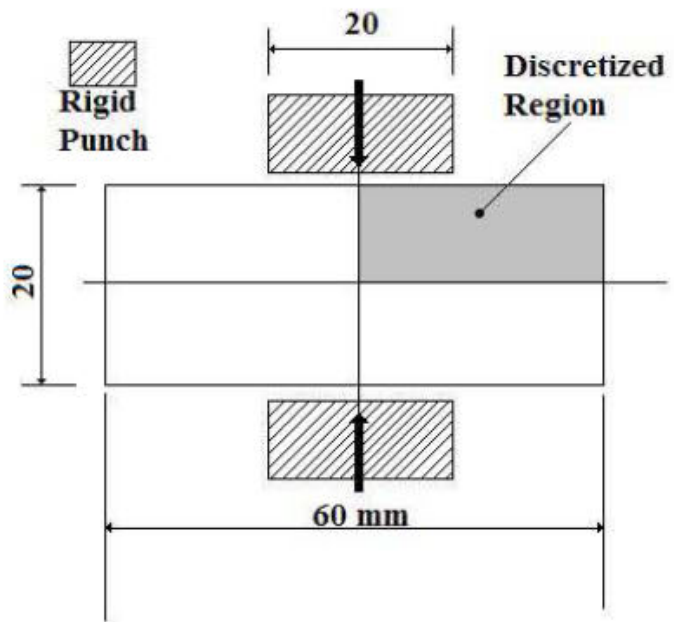

Figure 6. Schematic statement of the punch test.

Time $=2.5 \mathrm{e} 005 \mathrm{~s}$

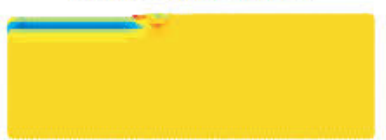

Time $=0.0001 \mathrm{~s}$

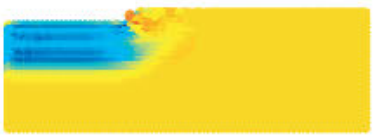

Time $=0.000175 \mathrm{~s}$

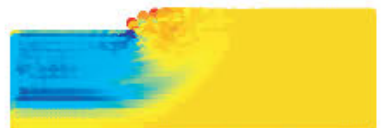

Time $=0.00025 \mathrm{~s}$

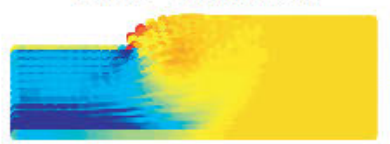

Time $=0.000325 \mathrm{~s}$

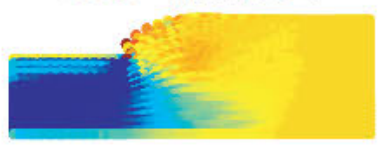

Time $=0.0004 \mathrm{~s}$

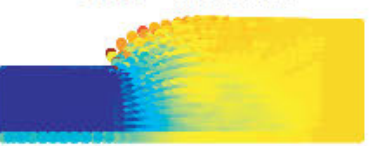

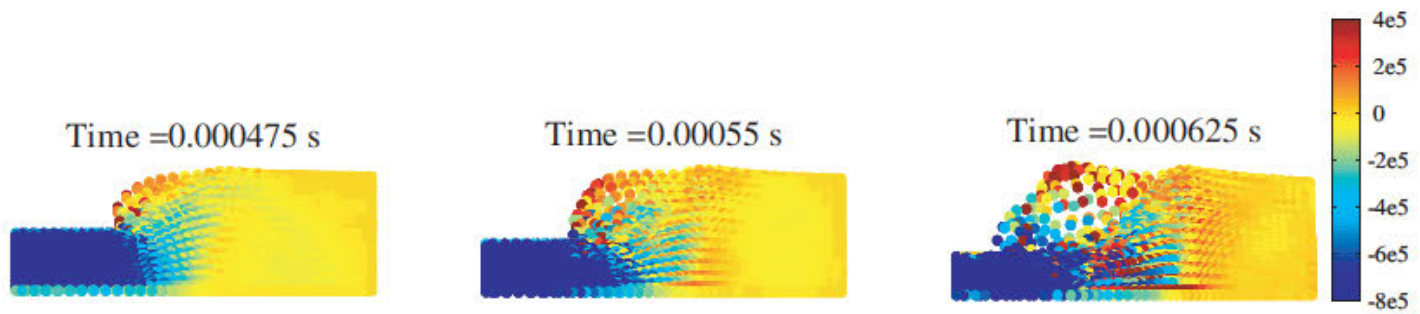

Figure 7. Total Lagrangian formulation: punch test. Stress component $\sigma_{z z}$ ( $z$ is the height component) is represented. 


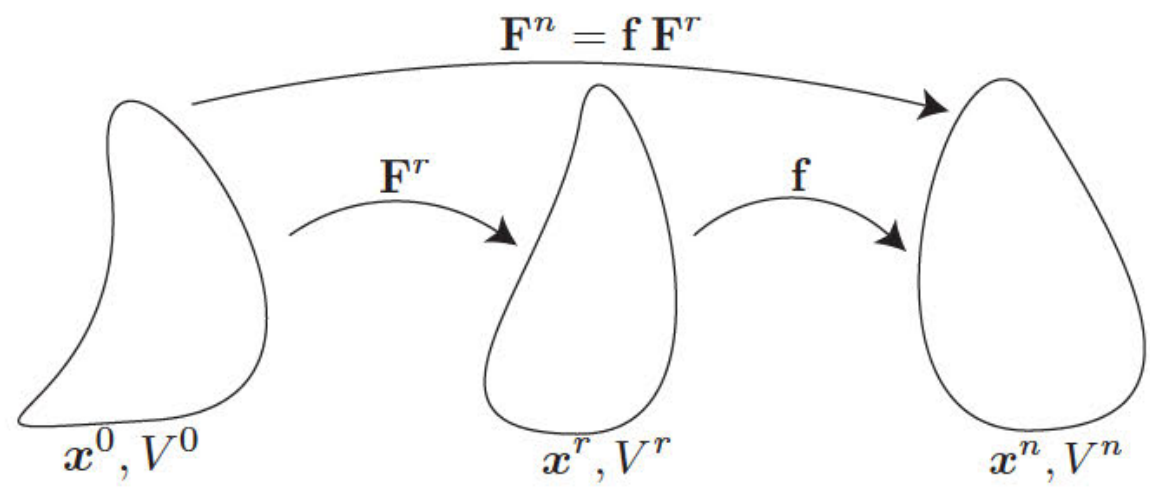

Figure 8. Updated Lagrangian.

Recalling the identity $\mathbf{A}: \mathbf{B}=\operatorname{tr}\left(\mathbf{A B}^{\mathrm{T}}\right)$, which implies that

$$
\mathbf{P}_{j}^{n}:\left(\delta \dot{\mathbf{f}}_{j} \mathbf{F}_{j}^{r}\right)=\operatorname{tr}\left(\mathbf{P}_{j}^{n}\left[\mathbf{F}_{j}^{r}\right]^{\top} \delta \dot{\mathbf{f}}_{j}^{\top}\right)=\left(\mathbf{P}_{j}^{n}\left[\mathbf{F}_{j}^{r}\right]^{\top}\right): \delta \dot{\mathbf{f}}_{j},
$$

the internal virtual work can be expressed as

$$
\delta \dot{w}_{\text {int }}=\sum_{j} V_{j}^{0}\left(\mathbf{P}_{j}^{n}\left[\mathbf{F}_{j}^{r}\right]^{\top}\right): \delta \dot{\mathbf{f}}_{j}=\sum_{j} V_{j}^{0}\left(\mathbf{P}_{j}^{n}\left[\mathbf{F}_{j}^{r}\right]^{\top}\right):\left(\sum_{k} \delta \mathbf{v}_{k} \boldsymbol{g}_{k}^{\top}\left(\boldsymbol{x}_{j}^{r}\right)\right),
$$

which can be further simplified using $\mathbf{A}:\left(\mathbf{u} \mathbf{v}^{\boldsymbol{\top}}\right)=\mathbf{u} \cdot \mathbf{A v}$ as

$$
\delta \dot{w}_{\text {int }}=\sum_{k} \delta \mathbf{v}_{k} \cdot\left(\sum_{j} V_{j}^{0}\left(\mathbf{P}_{j}^{n}\left[\mathbf{F}_{j}^{r}\right]^{\top}\right) g_{k}\left(\boldsymbol{x}_{j}^{r}\right)\right)
$$

and therefore at the current configuration, $x^{n}$, the internal forces vector at a given particle $i$ can be easily evaluated using a reference configuration $x^{r}$ as

$$
\mathbf{T}_{i}^{n, r}=\sum_{j} V_{j}^{0}\left(\mathbf{P}_{j}^{n}\left[\mathbf{F}_{j}^{r}\right]^{\mathrm{T}}\right) \boldsymbol{g}_{i}\left(x_{j}^{r}\right) .
$$

Remark 1. When an update is performed the mass and the density conservation are imposed and the weights of the numerical quadrature (also called volumes) are changed. In practice, the volume change is computed using the Jacobian $J$, see (2), as $V=J V^{0}$. Then, the element mass can be related to the volume element in terms of the initial and current densities as $d m=\zeta_{0} d V^{0}=\zeta d V$. Thus, conservation of mass is ensured and can be expressed as $\zeta_{0}=\zeta J$.

\section{2. $1 D$ analytical stability analysis}

An analytical stability analysis for the updated Lagrangian formulation is presented in this section. Consider a $1 \mathrm{D}$ bar discretized by a given number of particles which deforms from reference to final configurations as shown in Figure 9. Note that in the case of an updated Lagrangian corrected SPH formulation, the kernel functions are fixed at the reference configuration, $\boldsymbol{x}^{r}$. For simplicity particle spacing will be assumed to be uniform precisely at the reference configuration and only immediate neighbors of a given particle will contribute to 


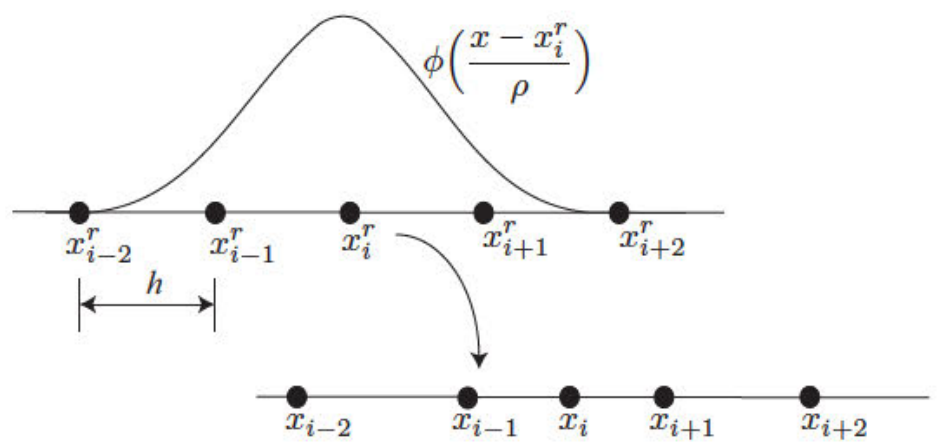

Figure 9. 1D Updated Lagrangian corrected SPH.

the internal force evaluation at this particle; that is, following reference [7] the kernel function centered at a given particle $i$ vanishes for any particle other than the next neighbors.

Given the one-dimensional nature of the problem, and imposing the area as a constant equal to one (in the reference configuration), the deformation gradient is simply given as

$$
\mathrm{F}_{i}=\mathrm{f}_{i} \mathrm{~F}_{i}^{r}=\left(\sum_{j} x_{j} g_{j}\left(x_{i}^{r}\right)\right) \mathrm{F}_{i}^{r} .
$$

For the simple uniformly spaced reference configuration considered, the linearly corrected gradient terms $g$ are simply

$$
g_{i-1}\left(x_{i}^{r}\right)=-\frac{1}{2 h}, g_{i}\left(x_{i}^{r}\right)=0, \text { and } g_{i+1}\left(x_{i}^{r}\right)=\frac{1}{2 h},
$$

which upon substitution into (15) leads to

$$
\mathrm{F}_{i}=\frac{x_{i+1}-x_{i-1}}{2 h} \mathrm{~F}_{i}^{r} .
$$

Consider now the internal force equation in the current $1 \mathrm{D}$ context

$$
\mathrm{T}_{i}^{n, r}=\sum_{j} V_{j}^{0} \mathrm{P}_{j} \mathrm{~F}_{j}^{r} g_{i}\left(x_{j}^{r}\right),
$$

which using equation (3) gives

$$
\mathrm{T}_{i}^{n, r}=\sum_{j} V_{j}^{n} \sigma_{j} g_{i}\left(x_{j}^{r}\right) / \mathrm{f}_{j} .
$$

The internal force at point $i$ is further developed substituting in the previous equation the gradient functions defined in (16), namely

$$
\mathrm{T}_{i}^{n, r}=\left(\frac{V_{i-1}^{n} \sigma_{i-1}}{\mathrm{f}_{i-1}}-\frac{V_{i+1}^{n} \sigma_{i+1}}{\mathrm{f}_{i+1}}\right) / 2 h .
$$

Using the linear constitutive relationship, $\sigma_{i}=\kappa\left(J_{i}-1\right)$ where $J_{i}$ is the Jacobian, see (2), at $x_{i}$, which in the one dimensional case is simply $J_{i}=\operatorname{det} \mathrm{f}_{i}=\mathrm{f}_{i}$, the internal force vector can be written as,

$$
\mathrm{T}_{i}^{n, r}=\frac{V_{i-1}^{n} \kappa-V_{i+1}^{n} \kappa}{2 h}+\frac{V_{i+1}^{n} \kappa}{\left(x_{i+2}-x_{i}\right)}-\frac{V_{i-1}^{n} \kappa}{\left(x_{i}-x_{i-2}\right)} .
$$




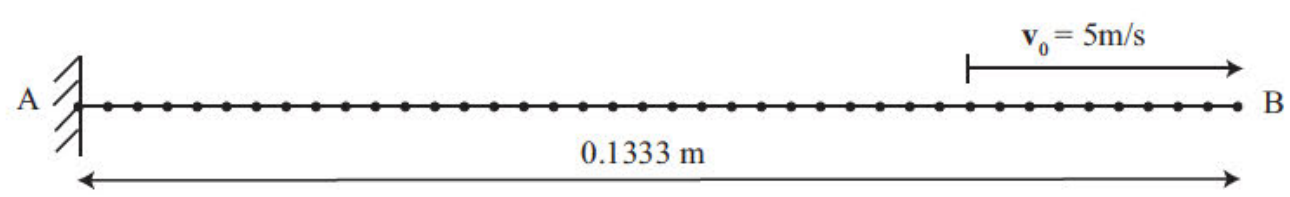

Figure 10. 1D bar problem statement.

Consequently, the tangent stiffness matrix terms are readily evaluated as

$$
\begin{gathered}
K_{i, i}=\frac{V_{i-1}^{n} \kappa}{\left(x_{i}-x_{i-2}\right)^{2}}+\frac{V_{i+1}^{n} \kappa}{\left(x_{i+2}-x_{i}\right)^{2}}, \\
K_{i, i+1}=K_{i, i-1}=0, \\
K_{i, i+2}=\frac{-V_{i+1}^{n} \kappa}{\left(x_{i+2}-x_{i}\right)^{2}}, \quad \text { and } \quad K_{i, i-2}=\frac{-V_{i-1}^{n} \kappa}{\left(x_{i}-x_{i-2}\right)^{2}} .
\end{gathered}
$$

Finally, a simple calculation shows that the alternating eigenvector $(-1)^{j}$ has an eigenvalue associated equal to zero, that is

$$
\sum_{j} K_{i j}(-1)^{j}=0 .
$$

The above equation implies that this alternating mode is a mechanism instead of a mode with a possible negative eigenvalue as is the case in the Eulerian formulation, see [16]. Consequently, the algorithm should be stable but, in the absence of artificial viscosity (that may be easily introduced by time integrators), undamped oscillations may emerge during the computations.

\subsection{Numerical examples}

3.3.1. 1D numerical tests The previous section has proven the existence of mechanisms in the updated Lagrangian formulation (as well as it was proven for the total Lagrangian in [16]). Next, a simple 1D numerical test is performed in order to verify whether the mechanisms are activated or not in a standard updated Lagrangian formulation.

The total Lagrangian and the updated Lagrangian formulations will be used to solve the elastic 1D bar problem described in Figure 10, see also [32]. The bar is fixed at the left end $\mathrm{A}$, free at the right end $\mathrm{B}$ and the right quarter of the bar is given an initial velocity of $\mathbf{v}_{0}=5 \mathrm{~m} / \mathrm{s}$ (as an initial condition) thus putting the bar in tension initially. Standard SPH methods cannot solve this problem due to tension instability that immediately develops.

The problem is solved using a uniform distribution of particles. As shown in Figure 10 the corrected SPH particle distribution is very coarse with only 40 uniform particles. Figure 11 presents the displacement time history of the right end B for the total Lagrangian formulation and the updated Lagrangian one with updates every 3 time steps. No artificial viscosity of any type is used. Figure 12 compares the predicted time history for the velocity of the right end $\mathrm{B}$ for the total and standard updated formulations. It becomes clear from Figures 11 and 12 that in the updated Lagrangian formulation the mechanisms are activated and they spoil the solution. It is clear that performing updates may activate the mechanisms, which spoil the solution. 

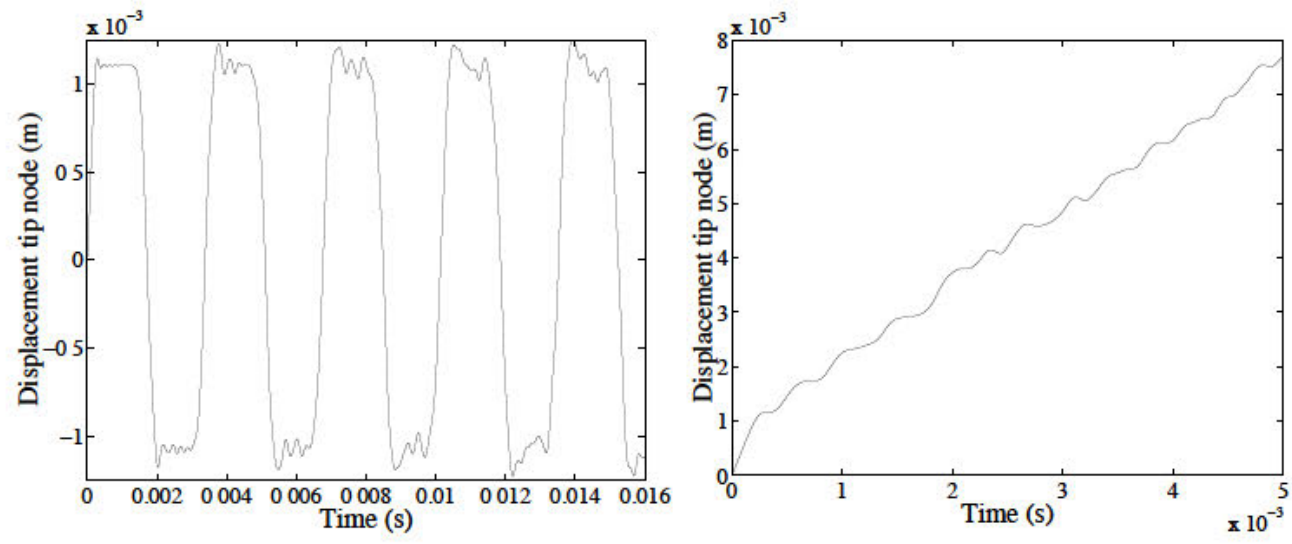

Figure 11. Displacement history for the right end of the bar (point B). Total Lagrangian (left) and updated Lagrangian (right) formulations.
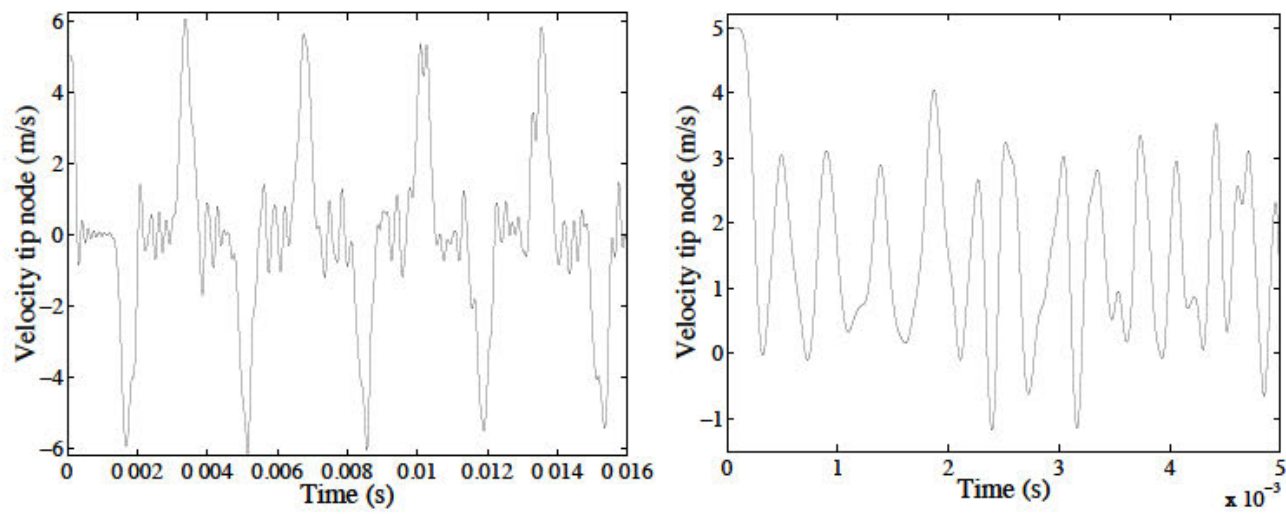

Figure 12. Velocity history for the right end of the bar (point B). Total Lagrangian (left) and updated Lagrangian (right) formulations.

3.3.2. Bending test and rubber rings collision The same behavior (zero energy modes activated by reevaluation of neighbors particles) can also be observed in the examples proposed in section 2.4.1 and 2.4.2. The standard updated Lagrangian formulation, with updates performed every three time-steps, activates the mechanisms as shown in Figures 13 and 14. It is important to note that such results are very similar to the well-known tension instabilities that appear in these examples if an Eulerian formulation is employed, cf. [20, 33]. However, the stability analysis showed that the updated formulation has zero-energy mechanisms but does not present modes with negative eigenvalues (as is the case in the Eulerian formulation).

It seems that these mechanisms are excited in the case of an updated Lagrangian formulation but not in the total Lagrangian case. This is possibly due to the fact that the modes associated to these mechanisms change with the update of reference configuration and it is no longer possible to ensure that the velocity field in the body is orthogonal to this modes. 


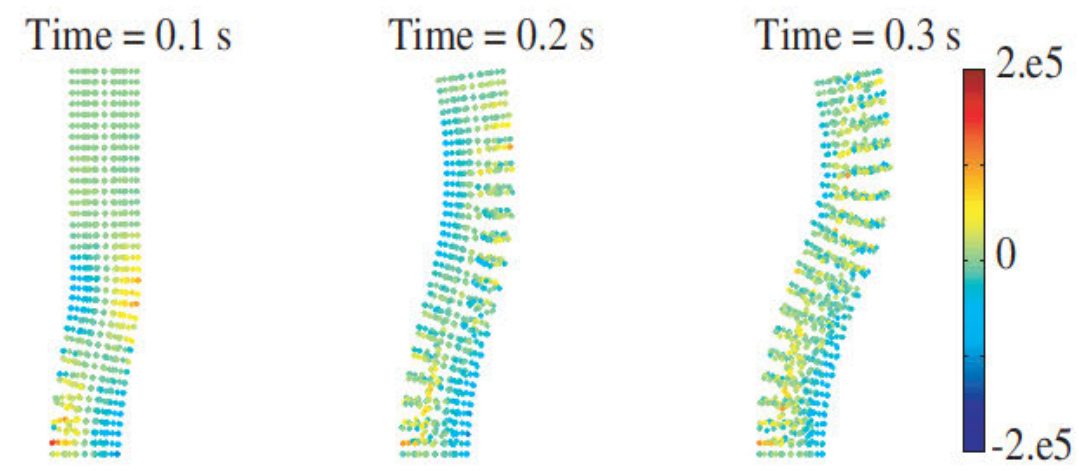

Figure 13. Standard updated Lagrangian formulation: cylinder bending test.

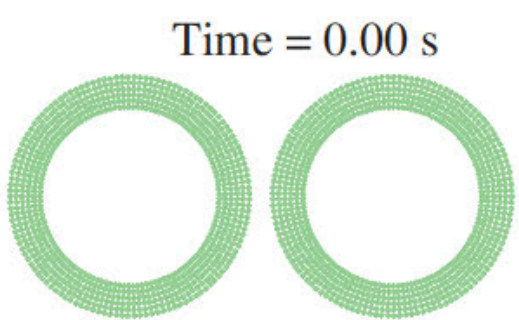

Time $=0.02 \mathrm{~s}$

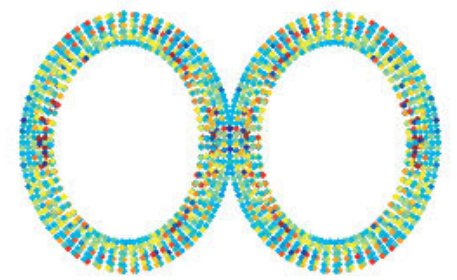

Time $=0.04 \mathrm{~s}$

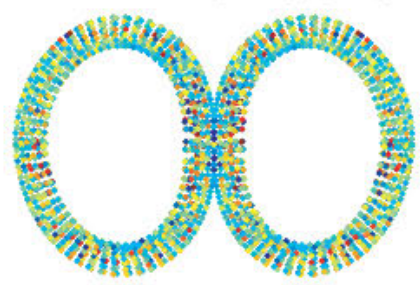

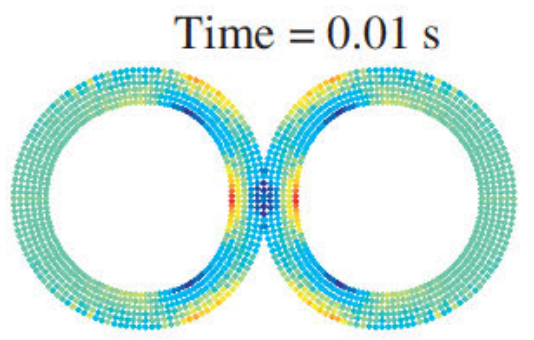

Time $=0.03 \mathrm{~s}$
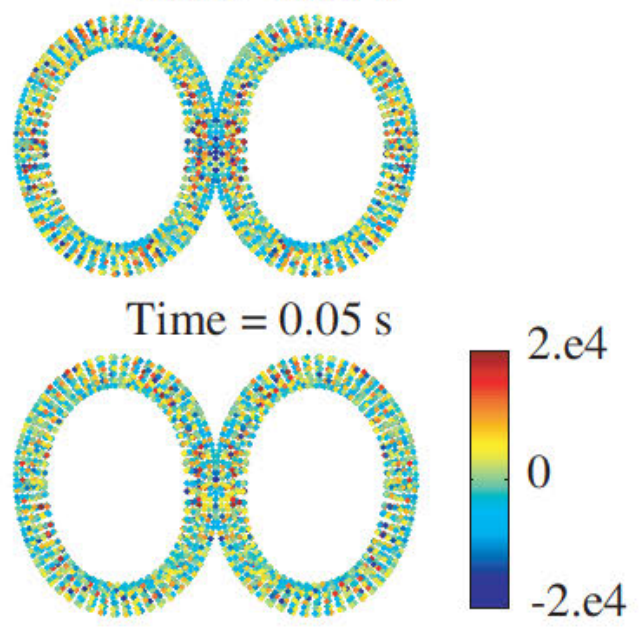

Figure 14. Standard updated Lagrangian formulation: rubber rings collision. 


\subsection{Hessian's Difference Stabilization}

Clearly, it is necessary to eliminate the observed mechanisms if an updated formulation is to be used. Next, a new stabilization technique is proposed. This method is based on the addition of a higher order derivative term to the corrected gradient of any function, $\psi$, which is approximated, see (6), with the standard expression:

$$
\psi(\boldsymbol{x})=\sum_{j} N_{j}(\boldsymbol{x}) \psi_{j}
$$

The added term is the difference of two Hessians which will converge to zero as the particle distribution is refined. Higher order derivatives have been extensively used to stabilize numerical computations, in particular, in the framework of SPH methods [13] introduces a Laplacian to stabilize a CSPH algorithm. In this way, the gradient of an arbitrary function $\psi$ is now evaluated as,

$$
\widetilde{\nabla}^{s} \psi\left(\boldsymbol{x}_{i}^{r}\right):=\sum_{j} \psi_{j} \boldsymbol{g}_{j}\left(\boldsymbol{x}_{i}^{r}\right)+\eta\left[\boldsymbol{H}_{\psi}\left(\boldsymbol{x}_{i}^{r}\right)-\nabla\left(\boldsymbol{\nabla} \psi\left(\boldsymbol{x}_{i}^{r}\right)\right)\right] \boldsymbol{h}
$$

where $\eta$ is a dimensionless stabilization parameter, $\boldsymbol{h}$ is a vector proportional to the interparticle distance, and $\mathcal{H}_{\psi}\left(\boldsymbol{x}_{i}^{r}\right)$ represents the Hessian of $\psi$, which in the context of corrected SPH can be obtained as

$$
\mathcal{H}_{\psi}\left(\boldsymbol{x}_{i}^{r}\right):=\sum_{k} V_{k}^{r} \psi_{k} \widetilde{\mathcal{H}}_{\phi_{k}}\left(\boldsymbol{x}_{i}^{r}\right)
$$

where $\widetilde{\mathcal{H}}_{\phi_{k}}$ is the linearly corrected Hessian kernel. To obtain linear reproducibility, $\widetilde{\mathcal{H}}_{\phi_{k}}$ is corrected by means of two terms, namely a matrix $\mathcal{B}(\boldsymbol{x})$ and a third order tensor $\mathcal{A}(\boldsymbol{x})$ as,

$$
\widetilde{\mathcal{H}}_{\phi_{k}}\left(\boldsymbol{x}_{i}^{r}\right)=\mathcal{H}_{\phi_{k}}\left(\boldsymbol{x}_{i}^{r}\right)+\delta_{i k} \mathcal{B}\left(\boldsymbol{x}_{i}^{r}\right)+\mathcal{A}\left(\boldsymbol{x}_{i}^{r}\right) \cdot\left(\boldsymbol{x}_{i}^{r}-\boldsymbol{x}_{k}^{r}\right),
$$

where $\boldsymbol{\mathcal { H }}_{\phi_{k}}\left(\boldsymbol{x}_{i}^{r}\right)$ is the Hessian of the kernel function $\phi$, that is,

$$
\mathcal{H}_{\phi_{k}}(\boldsymbol{x})=\nabla\left(\nabla \phi\left(\frac{\boldsymbol{x}-\boldsymbol{x}_{k}}{\rho}\right)\right),
$$

which has an explicit known expression once the kernel $\phi$ is defined. Correction terms $\mathcal{B}$ (second order tensor) and $\mathcal{A}$ (third order tensor) are determined enforcing that constant and linear functions must have null Hessian, that is,

$$
\sum_{k} V_{k}^{r} \widetilde{\mathcal{H}}_{\phi_{k}}\left(\boldsymbol{x}_{i}^{r}\right)=\mathbf{0} \text { and } \sum_{k} V_{k}^{r} \widetilde{\mathcal{H}}_{\phi_{k}}\left(\boldsymbol{x}_{i}^{r}\right) \boldsymbol{x}_{k}^{r}=\mathbf{0} .
$$

These reproducibility conditions determine the expressions for $\mathcal{B}(\boldsymbol{x})$ and $\mathcal{A}(\boldsymbol{x})$, namely

$$
\begin{gathered}
\mathcal{A}(\boldsymbol{x})=\left[\sum_{k} V_{k}^{r} \boldsymbol{\mathcal { H }}_{\phi_{k}}(\boldsymbol{x})\left(\boldsymbol{x}_{k}^{r}-\boldsymbol{x}\right)^{\top}\right] \cdot\left[\sum_{k} V_{k}^{r}\left(\boldsymbol{x}_{k}^{r}-\boldsymbol{x}\right)\left(\boldsymbol{x}_{k}^{r}-\boldsymbol{x}\right)^{\top}\right]^{-1} \\
\mathcal{B}(\boldsymbol{x})=\frac{1}{V_{i}^{r}}\left[-\sum_{k} V_{k}^{r} \boldsymbol{\mathcal { H }}_{\phi_{k}}(\boldsymbol{x})+V_{k}^{r} \mathcal{A}(\boldsymbol{x}) \cdot\left(\boldsymbol{x}_{k}^{r}-\boldsymbol{x}\right)\right]
\end{gathered}
$$



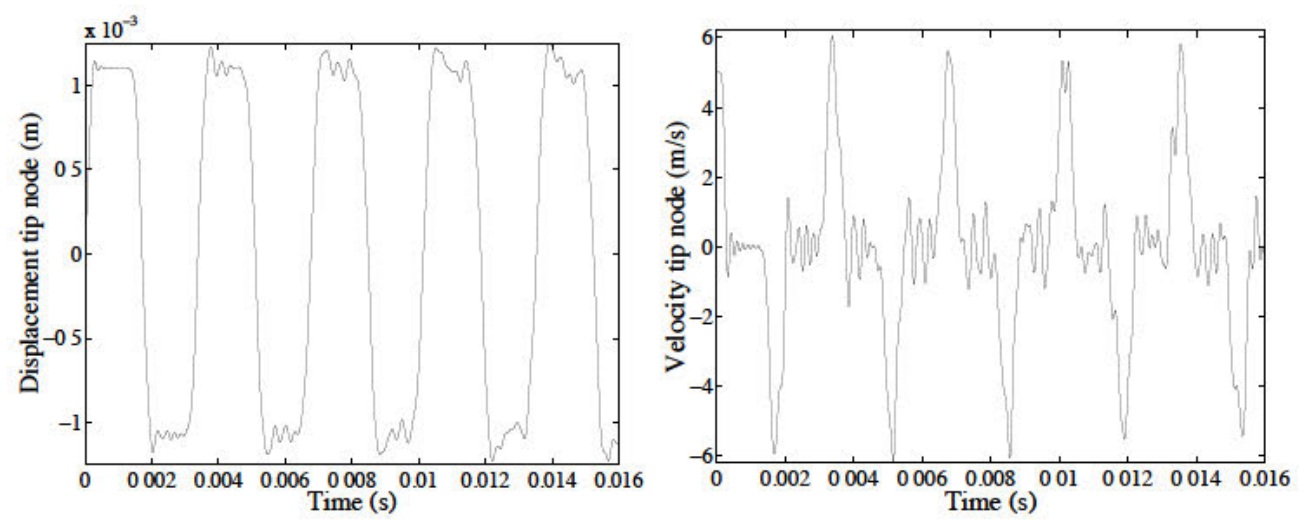

Figure 15. Displacement (left) and velocity (right) history for the right end of the bar using Hessian's difference stabilization.

Where in equation (19) the product of a third order tensor (in the first bracket) by a second order tensor (second bracket) is understood as

$$
[\mathcal{A}]_{\alpha \beta \delta}=[\mathcal{C}]_{\alpha \beta \gamma}\left[\mathcal{D}^{-1}\right]_{\gamma \delta}
$$

where $\alpha, \beta, \gamma$ and $\delta$ indicate spatial components. Equation (17) can be rewritten using the definition of the Hessian, equation (18), and the gradient, equation (13), as

$$
\sum_{k} \psi_{k} \widetilde{\boldsymbol{g}}_{k}^{s}(\boldsymbol{x})=\sum_{k} \psi_{k} \boldsymbol{g}_{k}(\boldsymbol{x})+\eta\left[\sum_{k} V_{k}^{r} \psi_{k} \widetilde{\mathcal{H}}_{\phi_{k}}(\boldsymbol{x})-\sum_{k} \psi_{k}\left(\sum_{l} \boldsymbol{g}_{k}\left(\boldsymbol{x}_{l}^{r}\right) \cdot \boldsymbol{g}_{l}^{\top}(\boldsymbol{x})\right)\right] \boldsymbol{h} .
$$

Hence $\widetilde{\boldsymbol{g}}_{k}^{s}(\boldsymbol{x})$ can be written as:

$$
\widetilde{\boldsymbol{g}}_{k}^{s}(\boldsymbol{x})=\boldsymbol{g}_{k}(\boldsymbol{x})+\eta\left[V_{k}^{r} \widetilde{\mathcal{H}}_{\phi_{k}}(\boldsymbol{x})-\left(\sum_{l} \boldsymbol{g}_{k}\left(\boldsymbol{x}_{l}^{r}\right) \boldsymbol{g}_{l}^{\top}(\boldsymbol{x})\right)\right] \boldsymbol{h},
$$

which is the expression that must be introduced, for instance, in (14) to evaluate the internal forces. Equation (21) represents the complete form for the corrected gradient of the kernel, it includes the correction (for reproducibility in the discrete setting —nodal integration-) and stabilization.

When this stabilization is introduced in the updated Lagrangian formulation the results for the one-dimensional bar problem presented in section 3.3.1 change drastically. In this case, the parameter $\eta$ is taken equal to 0.3 . Figure 15 shows both displacements and velocities for the right end of the bar, which now agree with the total Lagrangian results. It is important to observe that in order to check the performance of this approach an update is done every three time-steps, which is obviously unnecessary but has been done to trigger possible instabilities.

Remark 2. Vector $\boldsymbol{h}^{T}=\left\{h_{x}, h_{y}, h_{z}\right\}$ is proportional to the interparticle distance because its components $h_{x}, h_{y}, h_{z}$ are precisely the smoothing lengths of the kernel function along the cartesian directions. Here in the examples shown $h_{x}=h_{y}=h_{z}$ and are constat during the simulation. 
Remark 3. The scalar parameter $\eta$ controls the amount of artificial viscosity introduced. As in other stabilization techniques a balance must be sought between the amount of stabilization and the accuracy.

\section{NUMERICAL EXAMPLES}

This section is devoted to show the performance of the stabilized updated Lagrangian method proposed. First, two large deformation benchmarks are analyzed; those where an Eulerian formulation shows instabilities and the total Lagrangian formulation reproduces the correct behavior. Then the punch test, where the total Lagrangian formulation presents instabilities, is computed.

\subsection{Bending test}

The problem presented in section 2.4.1 is solved using an updated Lagrangian stabilized formulation. This test is particularly demanding for both tension instability (not present in any updated formulation) and spurious mechanisms, that must be suppressed with the stabilization. Moreover, in order to further evaluate the performance of the stabilization, updates are activated every three time-steps. This will ensure that updates are not activating any mechanisms. Results for $\eta=1.5$ can be seen in Figure 16 . Time $t=0.9 \mathrm{~s}$ is achieved with 8633 time steps. Thus, 2877 updates have been performed. Nevertheless, the cylinder deformation is in good agreement with to the total Lagrangian results. Figure 17 compares the results of the total Lagrangian and the stabilized updated formulation. It shows the maximum displacement history of the cylinder in the movement direction. Results for the stabilized updated formulation are almost superimposed with those of the total Lagrangian. Note that the artificial viscosity introduced by the stabilization only produces small phase lag but no appreciable decrease in amplitude. Figure 18 shows the stress component $\sigma_{z z}$ for the point in the bottom where tensile stresses develop first. Again, compared to the total Lagrangian solution, only a small phase lag is introduced by the stabilization.

\subsection{Rubber rings collision}

The problem presented in section 2.4.2 is solved. Updates are also performed every three timesteps. Results for $\eta=0.3$ can be seen in Figure 19. Time $t=0.05 \mathrm{~s}$ is achieved with 1333 time steps. Thus, 444 updates have been performed. Again, the stabilized updated formulation simulates the motion with no sign of fracture. Figure 20 compares the maximum displacement of the ring in the $y$-direction (which is perpendicular to the direction of initial velocity) of the total Lagrangian and the stabilized updated formulation. The results obtained using both methods are in good agreement. Figure 21 shows the stress history of the point in the top where tensile stresses develop first. Also in this example the artificial viscosity introduced is negligible.

\subsection{Punch test}

The total Lagrangian formulation was not able to solve the problem presented in section 2.4.3. This problem is solved again using the stabilized updated Lagrangian formulation. Updates 


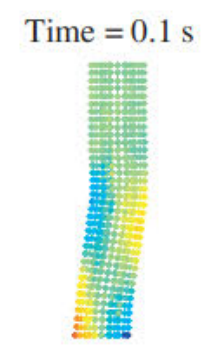

Time $=0.4 \mathrm{~s}$

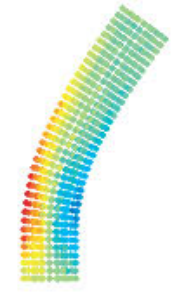

Time $=0.7 \mathrm{~s}$

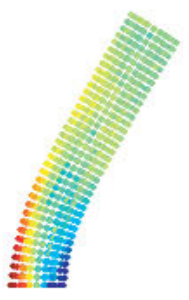

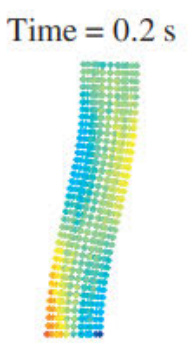

Time $=0.5 \mathrm{~s}$

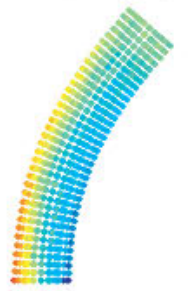

Time $=0.8 \mathrm{~s}$

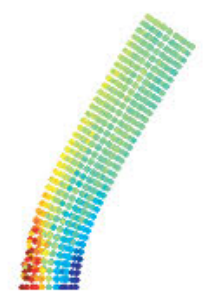

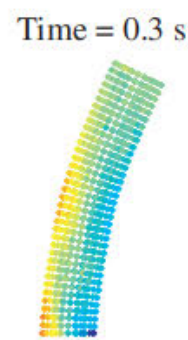

Time $=0.6 \mathrm{~s}$

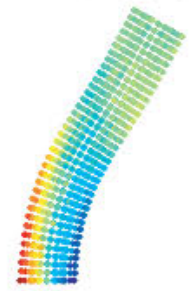

Time $=0.9 \mathrm{~s}$

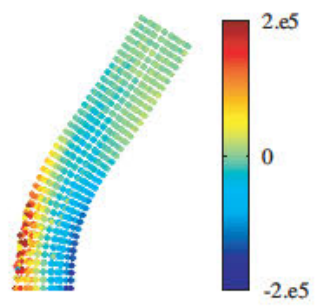

Figure 16. Stabilized updated Lagrangian formulation: cylinder bending test.

are also performed every three time-steps. Results for $\eta=1.4$ can be seen in Figure 22. The improved method is capable of solving this problem which involves extremely large distortions.

Obviously more updates than necessary are performed. In fact, in this example numerical experiments have shown that it is possible to decrease the number of updates to one every 10 steps without qualitative deterioration of the results. But, if an update is performed every 20 steps oscillations similar those observed with a total Lagrangian formulation are also obtained. An adaptive procedure that activates updates when large distortions are present is probably the best solution in this case to optimize the computational cost. Further research is needed in this area.

\section{CONCLUSIONS}

In this paper updated Lagrangian formulations in the SPH context have been studied. When large distortions are present the total Lagrangian formulation induces unacceptable results. 


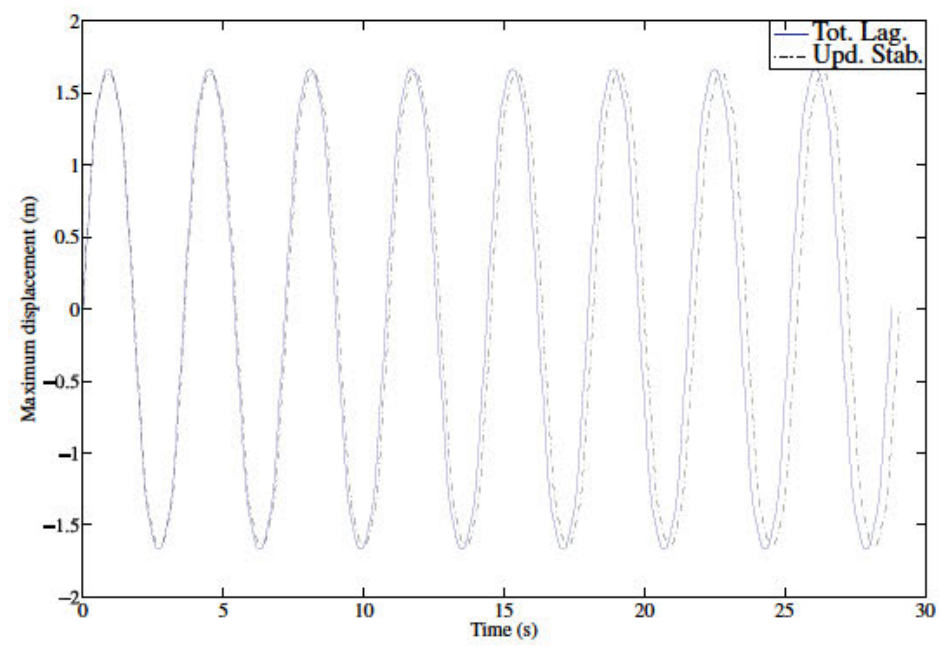

Figure 17. Maximum displacement history of the cylinder in the $x$-direction.

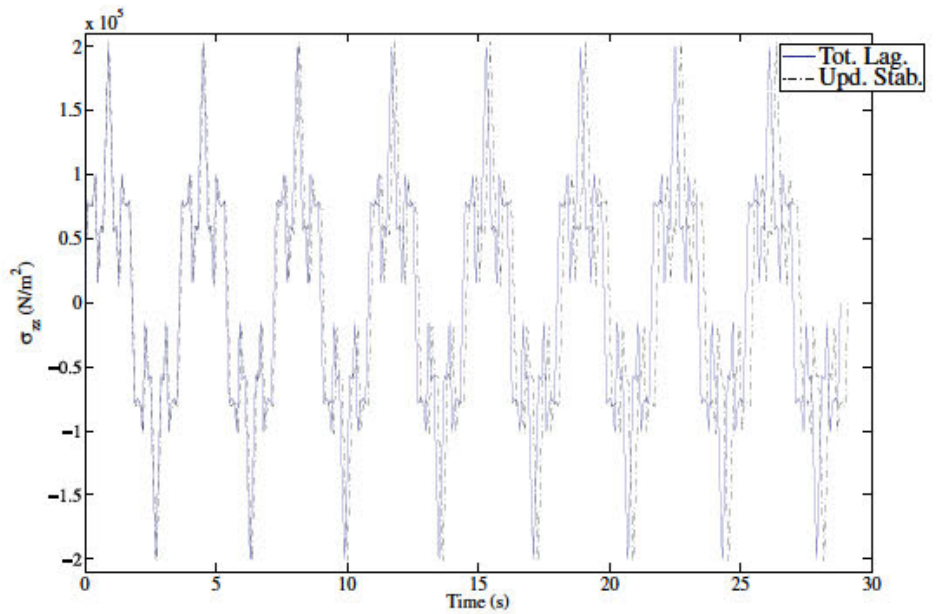

Figure 18. Stress history of the point in the bottom where tensile stresses develop first.

An updated Lagrangian formulation is then needed. That is, at some instances the neighboring particles should be recomputed.

Nevertheless, it is shown here that the standard updated formulation suffers the presence of zero energy modes. This formulation does not suffer from tensile instability. However, as shown in the examples these zero energy modes may be activated when updates are performed and the solution is then completely spoiled. Obviously, these mode may remain unnoticed if uncontrolled artificial viscosity is introduced by time integration for instance.

Here a consistent stabilized updated Lagrangian formulation has been proposed. Its behavior has been tested in benchmark tests for tensile instability, for zero energy modes and also for large distortions problems. The stabilized updated Lagrangian formulation behaves similarly 


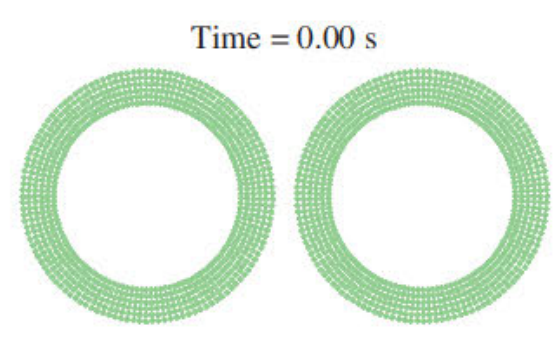

Time $=0.02 \mathrm{~s}$

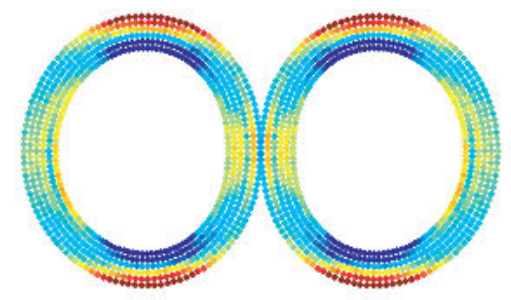

Time $=0.04 \mathrm{~s}$

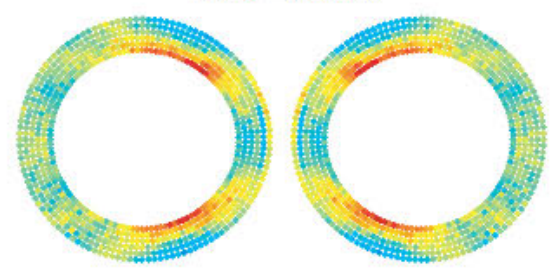

Time $=0.01 \mathrm{~s}$

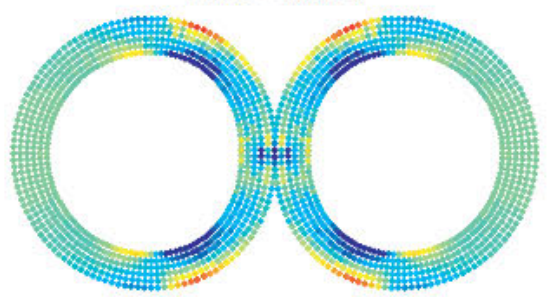

Time $=0.03 \mathrm{~s}$

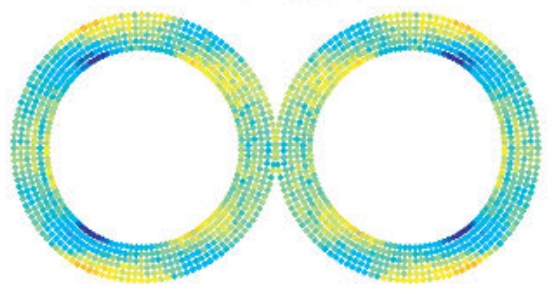

Time $=0.05 \mathrm{~s}$
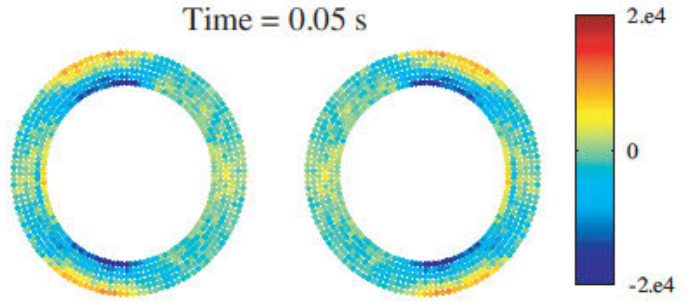

Figure 19. Stabilized updated Lagrangian formulation: collision of two rubber rings.

to the total Lagrangian formulation but can encompass problems involving large distortions using updates for the neighbors. Updates are only performed when needed; thus, in practice, the updated formulation has a low computational cost compared to the total Lagrangian one. Moreover, numerical examples show that even for an unreasonable number of updates -inducing, in practice, to a formulation where neighbors are recomputed almost at each timestep - zero (or negative) energy modes are precluded.

\section{REFERENCES}

1. S. W. Attaway, M. W. Heinstein, and J. W. Swegle, "Coupling of Smoothed Particle Hydrodynamics with the Finite Element Method," Nucl. Eng. Des., vol. 150, no. 2-3, pp. 199-205, 1994.

2. T. Belytschko, D. Organ, and Y. Krongauz, "A coupled finite element-element-free Galerkin method," Comput. Mech., vol. 17, no. 3, pp. 186-195, 1995.

3. G. R. Johnson, R. A. Stryk, and S. R. Beissel, "SPH for high velocity impact computations," Comput. Methods Appl. Mech. Eng., vol. 139, no. 1-4, pp. 347-373, 1996.

4. W. K. Liu, R. A. Uras, and Y. Chen, "Enrichment of the finite element method with the reproducing kernel particle method," J. Appl. Mech., ASME, vol. 64, pp. 861-870, 1997.

5. A. Huerta and S. Fernandez-Mendez, "Enrichment and coupling of the finite element and meshless methods," Int. J. Numer. Methods Eng., vol. 48, no. 11, pp. 1615-1636, 2000. 


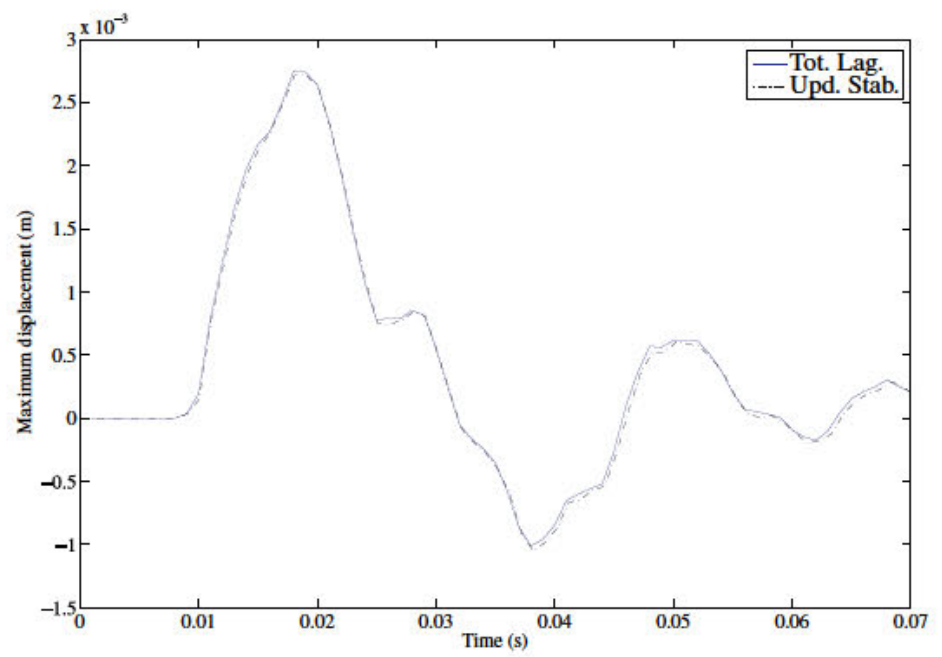

Figure 20. Maximum displacement history of the rubber rings in the $y$-direction.

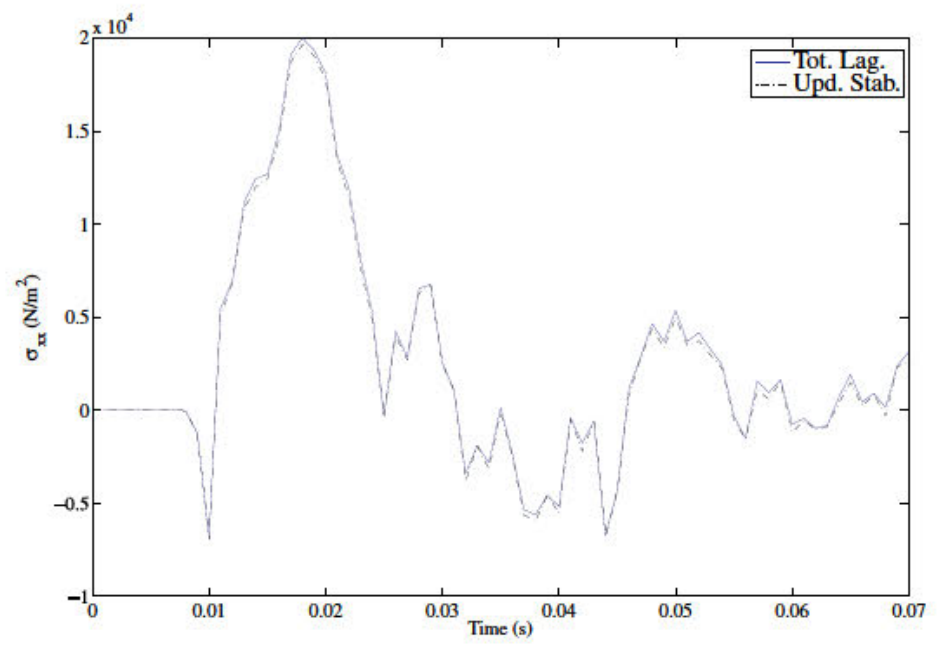

Figure 21. Stress history of the point in the top (or bottom as the solution has symmetries) where tensile stresses develop first.

6. S. Fernandez-Mendez, J. Bonet, and A. Huerta, "Continuous blending of SPH with finite elements," Comput. Struct., vol. 83, no. 17-18, pp. 1448-1458, 2005.

7. J. W. Swegle, D. L. Hicks, and S. W. Attaway, "Smoothed particle hydrodynamics stability analysis," J. Comput. Phys., vol. 116, no. 1, pp. 123-134, 1995.

8. T. Belytschko, Y. Guo, W. K. Liu, and S. P. Xiao, "A unified stability analysis of meshless particle methods," Internat. J. Numer. Methods Engrg., vol. 48, no. 9, pp. 1359-1400, 2000.

9. A. Huerta, T. Belytschko, S. Fernandez-Mendez, and T. Rabczuk, "Meshfree methods," in Encyclopedia of Computational Mechanics (E. Stein, R. de Borst, and T. J. R. Hughes, eds.), vol. 1 Fundamentals, ch. 10, pp. 279-309, Chichester: John Wiley \& Sons, Ltd., 2004.

10. M. B. Liu, G. R. Liu, and K. Y. Lam, "Constructing smoothing functions in smoothed particle hydrodynamics with applications," J. Comput. Appl. Math., vol. 155, no. 2, pp. 263-284, 2003. 
Time $=2.5 \mathrm{e} 005 \mathrm{~s}$

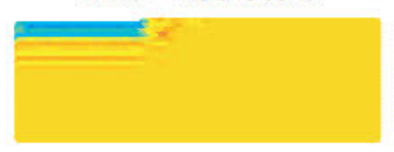

Time $=0.0001 \mathrm{~s}$

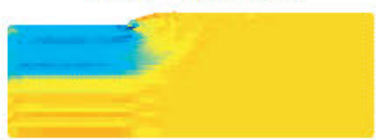

Time $=0.000175 \mathrm{~s}$

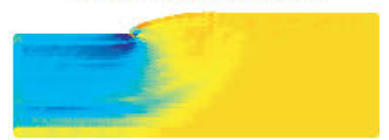

Time $=0.00025 \mathrm{~s}$

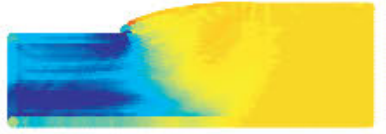

Time $=0.000475 \mathrm{~s}$

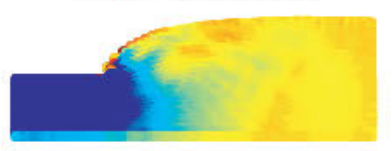

Time $=0.000325 \mathrm{~s}$

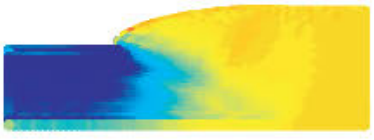

Time $=0.00055 \mathrm{~s}$

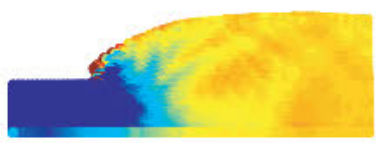

Time $=0.0004 \mathrm{~s}$

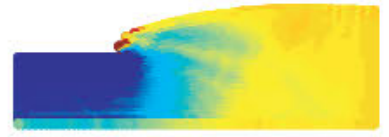

Figure 22. Stabilized updated Lagrangian formulation: punch test.

11. G. R. Johnson and S. R. Beissel, "Normalized smoothing functions for SPH impact computations," Int. J. Numer. Methods Eng., vol. 39, no. 16, pp. 2725-2741, 1996.

12. J. K. Chen, J. E. Beraun, and T. C. Carney, "A corrective smoothed particle method for boundary value problems in heat conduction," Int. J. Numer. Methods Eng., vol. 46, no. 2, pp. 231-252, 1999.

13. J. Bonet and S. Kulasegaram, "Correction and stabilization of smooth particle hydrodynamics methods with applications in metal forming simulations," Int. J. Numer. Methods Eng., vol. 47, no. 6, pp. 1189$1214,2000$.

14. J.-S. Chen, C.-T. Wu, S. Yoon, and Y. You, "A stabilized conforming nodal integration for galerkin mesh-free methods," Int. J. Numer. Methods Eng., vol. 50, no. 2, pp. 435-466, 2001.

15. J. J. Monaghan, "Why particle methods work," SIAM J. Sci. Stat. Comput., vol. 3, no. 4, pp. 422-433, 1982.

16. J. Bonet and S. Kulasegaram, "Remarks on tension instability of Eulerian and Lagrangian corrected smooth particle hydrodynamics (CSPH) methods," Int. J. Numer. Methods Eng., vol. 52, no. 11, pp. 1203$1220,2001$.

17. L. D. Libersky, A. G. Petschek, T. C. Carney, J. R. Hipp, and F. A. Allahdadi, "High strain Lagrangian hydrodynamics. A three-dimensional SPH code for dynamic material response," J. Comput. Phys., vol. 109, no. 1 , pp. $67-75,1993$.

18. G. R. Johnson, E. H. Petersen, and R. A. Stryk, "Incorporation of an SPH option into the EPIC code for a wide-range of high-velocity impact computations," Int. J. Impact Eng., vol. 14, no. 1-4, pp. 385-394, 1993.

19. R. Stellingwerf and C. Wingate, "Impact modeling with Smooth Particle Hydrodynamics," Int. J. Impact Eng., vol. 14, no. 1-4, pp. 707-718, 1993.

20. J. Gray, J. Monaghan, and R. Swift, "SPH elastic dynamics," Comput. Methods Appl. Mech. Eng., vol. 190, no. 49-50, pp. 6641-6662, 2001.

21. P. W. Randles and L. D. Libersky, "Normalized SPH with stress points," Int. J. Numer. Methods Eng., vol. 48 , no. 10, pp. 1445-1462, 1996. 
22. R. Vignjevic, J. Campbell, and L. Libersky, "A treatment of zero-energy modes in the smoothed particle hydrodynamics method," Comput. Methods Appl. Mech. Eng., vol. 184, no. 1, pp. 67-85, 2000.

23. J. Bonet and R. D. Wood, Nonlinear continuum mechanics for finite element analysis. Cambridge: Cambridge University Press, 1997.

24. L. Lucy, "Numerical approach to testing of the fission hypothesis," Astron. J., vol. 82, no. 12, pp. 10131024, 1977.

25. W. K. Liu, S. Jun, and Y. F. Zhang, "Reproducing kernel particle methods," Int. J. Numer. Methods Fluids, vol. 20, no. 8-9, pp. 1081-1106, 1995.

26. P. W. Randles and L. D. Libersky, "Smoothed particle hydrodynamics: some recent improvements and applications," Comput. Methods Appl. Mech. Eng., vol. 139, no. 1-4, pp. 375-408, 1996.

27. Y. Krongauz and T. Belytschko, "Consistent pseudo-derivatives in meshless methods," Comput. Methods Appl. Mech. Eng., vol. 146, no. 3-4, pp. 371-386, 1997.

28. J. P. Vila, "On particle weighted methods and smooth particle hydrodynamics," Math. Models Methods Appl. Sci., vol. 9, no. 2, pp. 161-209, 1999.

29. S. Fernandez-Mendez and A. Huerta, "Imposing essential boundary conditions in mesh-free methods," Comput. Methods Appl. Mech. Eng., vol. 193, no. 12-14, pp. 1257-1275, 2004.

30. A. Huerta and F. Casadei, "New ALE applications in non-linear fast-transient solid dynamics," Eng. Comput., vol. 11, no. 4, pp. 317-345, 1994.

31. A. Rodriguez-Ferran, F. Casadei, and A. Huerta, "ALE stress update for transient and quasistatic processes," Int. J. Numer. Methods Eng., vol. 43, no. 2, pp. 241-262, 1998.

32. C. T. Dyka and R. P. Ingel, "An approach for tension instability in smoothed particle hydrodynamics (SPH)," Comput. Struct., vol. 57, no. 4, pp. 573-580, 1995.

33. T. Belytschko and S. Xiao, "Stability analysis of particle methods with corrected derivatives," Comput. Math. Appl., vol. 43, no. 3-5, pp. 329-350, 2002. Radial basis functions and partial differential equations. 